\title{
Structural Health Monitoring of Composite Structures with Piezoelectric-Wafer Active Sensors
}

\author{
Victor Giurgiutiu* and Giola Santoni-Bottai $\stackrel{ \pm}{ }$ \\ University of South Carolina, Columbia, South Carolina 29208
}

DOI: $10.2514 / 1 . J 050641$

\begin{abstract}
Piezoelectric wafer active sensors are lightweight and inexpensive enablers for a large class of structural health monitoring applications such as 1) embedded guided-wave ultrasonics, i.e., pitch-catch, pulse-echo, and phased arrays; 2) high-frequency modal sensing, i.e., the electromechanical impedance method; and 3) passive detection (acoustic emission and impact detection). The focus of this paper will be on the challenges and opportunities posed by use of piezoelectric-wafer active sensors for structural health monitoring of composite structures as different from that of the metallic structures on which this methodology was initially developed. After a brief introduction, the paper discusses damage modes in composites. Then it reviews the structural health monitoring principles based on piezoelectric-wafer active sensors. This is followed by a discussion of guided-wave propagation in composites and how piezoelectric-wafer active sensor tuning can be achieved. Finally, the paper presents some damage detection results in composites: 1) hole damage in unidirectional and quasi-isotropic plates and 2) impact damage in quasiisotropic plates. The paper ends with conclusions and suggestions for further work.
\end{abstract}

$A$

Q3 $\quad A_{k}$

$A_{k}$

Q4

\section{Nomenclature \\ $=$ overall transfer matrix for the composite laminate \\ $=$ transfer matrix for the $k$ th layer in the TM method}

$=$ amplitude of the partial waves in the $k$ th layer in the global matrix method

$=$ antisymmetric Lamb-wave modes

$=$ half-length of the piezoelectric-wafer active sensors, $m$

$=$ electrical capacitance, $\mathrm{F}$

$C$
$C$

$C^{\prime}$

$c$

$c_{E}$

$c_{g}$

$c_{s}$

$[D]$

$\begin{array}{ll}D_{j} & =\text { electrical displacement tensor, } \mathrm{C} / \mathrm{m}^{2} \\ d & =\text { half-thickness, } \mathrm{m} \\ d_{k i j} & =\text { piezoelectric constant, } \mathrm{m} / \mathrm{V} \\ E_{k} & =\text { electrical field tensor, } \mathrm{V} / \mathrm{m} \\ E_{1} & =\text { axial modulus, } \mathrm{Pa} \\ E_{2} & =\text { transverse modulus, } \mathrm{Pa} \\ f & =\text { frequency, } \mathrm{Hz} \\ G_{12}, G_{23} & =\text { shear moduli, } \mathrm{Pa} \\ \mathrm{Im} & =\text { imaginary part of a complex quantity } \\ i & =\sqrt{-1}\end{array}$

Presented as Paper 2010-2661 at the 51st AIAA/ASME/ASCE/AHS/ASC Structures, Structural Dynamics, and Materials Conference, Orlando, FL, 12 April 2008-15 April 2010; received 10 May 2010; revision received 22 October 2010; accepted for publication 26 October 2010. Copyright (C 2010 by Victor Giurgiutiu. Published by the American Institute of Aeronautics and Astronautics, Inc., with permission. Copies of this paper may be made for personal or internal use, on condition that the copier pay the $\$ 10.00$ per-copy fee to the Copyright Clearance Center, Inc., 222 Rosewood Drive, Danvers, MA 01923; include the code and $\$ 10.00$ in correspondence with the CCC.

Q1 *Professor, Department of Mechanical Engineering, 300 Main Street South. Associate Fellow AIAA.

'Postdoctoral Research Fellow, Department of Mechanical Engineering, 300 Main Street South.

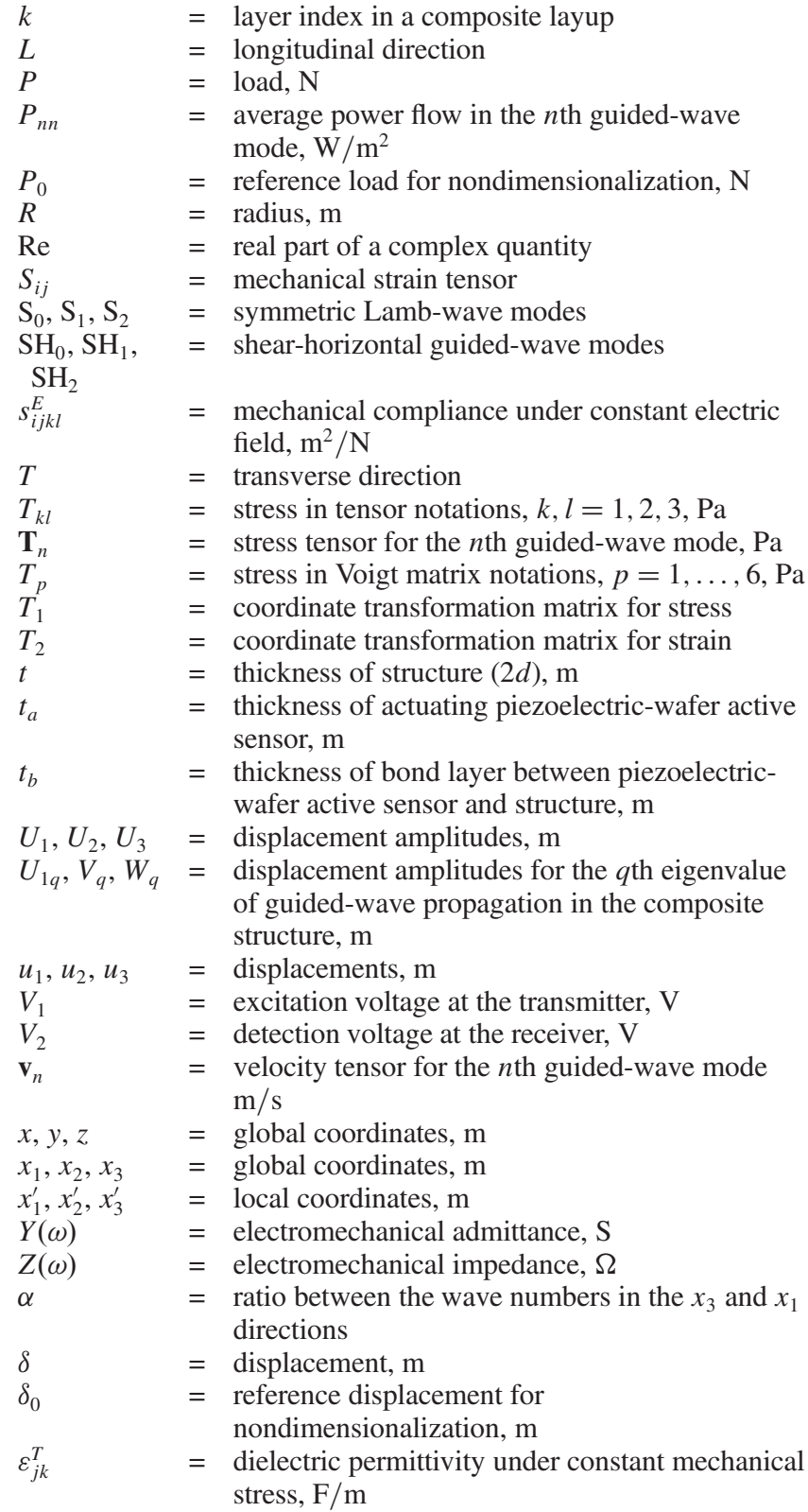


$\theta$

$\kappa_{31}$

$v_{12}, v_{23}$

$\xi$

$\omega$

$=$ wave-propagation angle with respect to the fiber direction

$=$ electromechanical coupling coefficient

$=$ Poisson's ratios

$=$ wave number, $1 / \mathrm{m}$

$=$ angular frequency, $\mathrm{rad} / \mathrm{s}$

\section{Introduction}

$\mathbf{S}$ TRUCTURAL health monitoring (SHM) is an emerging technology with multiple applications in the evaluation of critical structures. The goal of SHM research is to develop a monitoring methodology that is capable of detecting and identifying, with minimal human intervention, various damage types during the service life of the structure. Numerous approaches have been used in recent years to perform structural health monitoring [1,2]; they can be broadly classified into two categories: passive methods and active methods. Passive SHM methods (such as acoustic emission, impact detection, strain measurement, etc.) have been studied longer and are relatively mature; however, they suffer from several drawbacks that limit their utility (need for continuous monitoring, indirect inference of damage existence, etc.). Active SHM methods are currently of greater interest, due to their ability to interrogate a structure at will. One of the promising active SHM methods uses arrays of piezoelectric-wafer active sensors (PWASs) bonded to a structure for both transmitting and receiving ultrasonic waves in order to achieve damage detection [3].

There has been a marked increase in recent years in the use of composite materials in numerous types of structures, particularly in air and spacecraft structures. Composites have gained popularity in high-performance products that need to be lightweight, yet strong enough to take high loads such as aerospace structures, space launchers, satellites, and racing cars. Their growing use has arisen from their high specific strength and stiffness, when compared with metals, and the ability to shape and tailor their structure to produce more aerodynamically efficient configurations [4].

For this reason, it is important to study how active SHM methods (which were initially developed for isotropic metallic structures) can be extended to reliably detecting damage in these new types of materials, which are multilayered and anisotropic. One of the most troubling forms of damage in laminar composites is low-velocity impact damage. This type of damage can leave no visual traces, but subsurface delaminations can significantly reduce the strength of the structure.

The present paper presents and discusses the challenges and opportunities related to the use of PWASs in generating and sensing ultrasonic guided waves in composite materials and how they can be used to detect damage in composite structures. The paper starts with a brief a presentation of the main composite materials damage types, which are often different from those encountered in metallic structures. Then, it reviews the principles of PWAS-based SHM. Subsequently, the paper discusses the analytical challenges of studying guided waves in composites and shows how the concept of guided-wave tuning with a PWAS can be applied in the case of composite structures: theoretical predictions and experimental tuning results are comparatively presented. Finally, the paper presents experimental results on detecting hole and impact damage in composite plates with PWAS-based pitch-catch and pulse-echo methods. Hole damage detection experiments were performed on unidirectional and quasi-isotropic plates, and impact damage detection experiments were performed only on quasi-isotropic plates. The paper ends with conclusions and suggestions for further work.

\section{Damage in Composite Materials}

Composite materials combine the properties of two or more constituent materials in order to achieve properties that are not achievable by the individual constituents. For example, carbon-fiber composites combine the extreme specific stiffness and strength of carbon fibers with the binding properties of a polymeric matrix. In general, composites can be created from any two materials (metals, ceramics, polymers, elastomers, and glasses) that could be mixed in many ways (particulate inclusions, chopped-fiber, woven, unidirectional fibers, etc.); the final composite properties vary with the choice of constituents and the composite architecture. For high-performance structural applications, laminate composites made up of highstrength/high-stiffness unidirectional layers stacked at various angles have gained wide application. The choice of the orientation angles in the stacking sequence allows tailoring of the composite properties along certain preferential directions that are expected to experience highest operational loads. The simplest layup sequence is the $0 / 90$ (cross-ply) laminate, which consists of alternating 0 and 90 deg layers (Fig. 1). Some layup sequences such as 0/45/90 and 0/60/120 are dubbed quasi-isotropic, because they try to equalize the effective properties by applying the fibers in several directions [5].

The damage and failure of metallic structures is relatively well understood; their in-service damage and failure occurs mostly due to fatigue cracks that propagate under cyclic loading in metallic material. In contrast, the damage of composite materials occurs in many more ways than that of metals [6]. Composites fail differently under tension than they fail in compression, and the effect of fastener holes is much more complicated than in metals. In addition, the composites are prone to hidden damage from low-velocity impact (e.g., the drop of a hand tool); such damage can be barely visible and
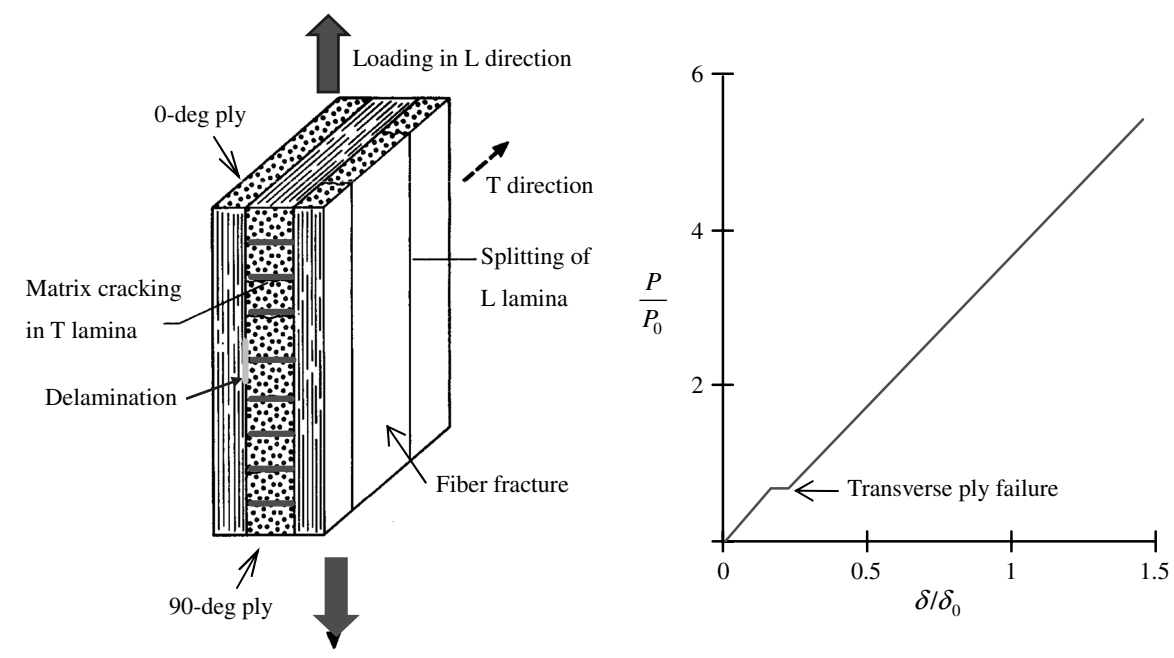

a)

b)

Fig. 1 Longitudinal tension of a 0/90 composite laminate: a) highlight of several damage modes: matrix cracking in transverse $T$ lamina, splitting of longitudinal $L$ laminas, and delamination between $T$ and $L$ laminas (after [ㅁ] $)$ and b) load/displacement curve showing kink and slope change after transverse ply failure. 
may go undetected, but its effect on the degradation of the composite structure strength can be dramatic. These various damage aspects will be discussed briefly in the next sections.

Current design requirements for composite structures are much more stringent than for metallic structures. Military aircraft components have to comply with an Aircraft Structural Integrity Program (ASIP) following the JSSG-2006 [7] and MIL-STD-1530 [8] guidelines. Preexisting manufacturing flaws and service-induced cracking are assumed to exist, even if undetected, and the ASIP function is to manage this fact while preventing aircraft accidents and downtime. In general, metallic structures are allowed to exhibit a certain amount of subcritical crack growth within the design life of the component. Detectable cracks are noted and managed as part of the maintenance and inspection process. In contrast, no known delamination cracks are allowed to exist (much less grow) in composite structure. However, the composite components are generally designed to tolerate a certain size of undetectable damage. Of course, this additional safety margin comes with a weight penalty, which could be mitigated through better understanding of composite damage detection and management mechanisms.

To satisfy the damage tolerance requirements, one has to Q6demonstrate that an aircraft structure possesses adequate residual strength at the end of service life in the presence of an assumed worstcase damage as, for example, that caused by a low-velocity impact on a composite structure. This may be accomplished by showing positive margins of safety at the maximum recommended load. Worst-case damage is defined as the damage caused by an impact event (e.g., a 1 in. hemispherical impactor) at the lesser of the following two energy levels: $100 \mathrm{ft} \cdot \mathrm{lb}$ or the energy to cause a visible dent ( $0.1 \mathrm{in}$. deep).

\section{A. Tension Damage in Composite Materials}

When subjected to axial tension, the composite material displays progressive failure through several damage mechanisms take place sequentially. Consider, for example, the cross-ply laminate of Fig. 1: as an axial load is applied in the longitudinal $L$ direction, the 0 deg ply is loaded along its reinforcing fibers, and the $90 \mathrm{deg}$ ply is loaded across the fibers. Because the strength of the polymeric matrix is much less than that of the fibers, the across-the-fiber strength of the lamina is much lower than the along-the-fiber strength. Hence, matrix cracking of the 90 deg ply occurs at an early stage in the loading cycle (Fig. 1b). As the tension load increases, further damage occurs in the form of delaminations between the 0 and 90 deg plies, due to 3-D effects at the interface between these two plies with such radically different properties. The matrix cracks existing in the transverse ply act as discontinuities generating 3-D disbonding stress that promotes delaminations. Same 3-D effects will lead to splitting of the 0 deg plies at higher tension loads. If the load continues to increase, the 0 deg plies will eventually fail due to fiber fracture, at which point no load can be supported any longer [9].

This simple 0/90 example indicates that internal damage in a composite laminate can happen at relatively low stress levels in the form of matrix cracking to be followed, at intermediate levels, by interply delamination and lamina splitting. If the applied stress is cyclic, as in fatigue loading, then these low-level damage states can increase and propagate further and further into the composite with each load cycle. The reinforcing fibers have high strength and good load-carrying properties, but the matrix cracking, delamination, and lamina splitting mechanisms usually lead to in-service composite structures becoming operationally unfit and requiring replacement.

\section{B. Compression Damage in Composite Structures}

When subjected to axial compression, the composite fails through loss of elastic stability (buckling). At the global scale, buckling can be avoided by relative sizing the length and bending stiffness of the component such that loss of elastic stability does not occur for the given boundary conditions and operational load levels. At the local scale, the composite material itself can fail under compression through the microbuckling mechanism (Fig. 2). The high-strength fibers encased in the polymeric matrix can be modeled as beams on an elastic foundation, where the elastic support is provided by the matrix stiffness. Under axial compression, such a beam on an elastic foundation would eventually buckle and take an undulatory shape (Fig. 2a). The compressive stress values at which this buckling occurs are dictated by the fiber bending stiffness and matrix compression stiffness. For a given fiber/matrix combination, this microbuckling compressive strength is fixed and cannot be altered through structural design. (Thicker fibers, e.g., boron fibers, have a higher compression buckling strength than the thinner carbon fibers; for this reason, boron composites may be preferred in places where material compression strength is critical.) As the compressive load is further increased, the microbuckling is further exacerbated until local failure occurs in the form of a kink band (Fig. 2b) [10].

\section{Fastener Hole Damage in Composite Structures}

Mechanical fasteners used in riveted and bolted joints are prevalent in metallic aircraft structures, where they offer a rapid and convenient method of assembling large structures from smaller components. The load-bearing mechanisms of metallic joints are well understood and easily predicted. The use of mechanical fasteners in composite structures is also allowed, but this comes with significant strength and fatigue penalties. The use of mechanical fasteners in composite structures somehow clashes with the very nature of composite materials, which carry the load through the highstrength fibers embedded in a relatively-weak polymeric matrix. This type of load-carrying capability benefits from a smooth and continuous load flow and is adverse to sudden changes in material properties and geometries. Ideally, composite joints should be done through adhesive bonding with gradual transition from one component into the next. However, when mechanical fasteners are used, they produce sudden discontinuities in the form of holes drilled in the composite structures; these fastener holes act as stress concentrators. They also act as crack and delamination initiators, due to microdamage introduced during the hole-drilling process. For this reason, mechanical fasteners do not seem appropriate for composite construction unless special attention is given to creating stress-free

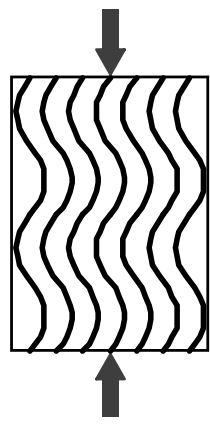

a)

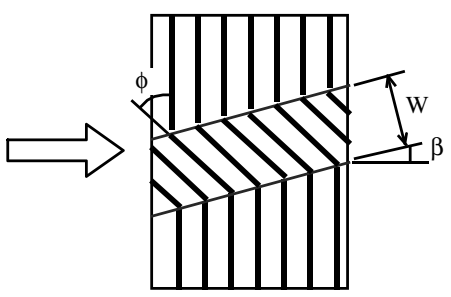

b)

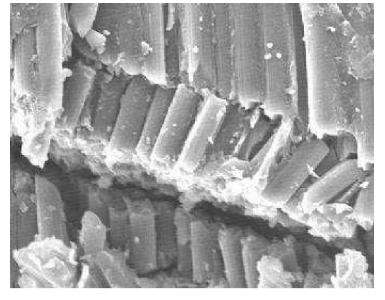

c)

Fig. 2 Compression damage of fiber composites through microbuckling: a) undulations of buckled fibers; b) kink band local failure schematic; c) micrograph of kink band formation in T800/924C carbon-fiber composite (after [8]). 
holes during design through local reinforcement and damage-free holes during manufacturing through special tooling. In spite of these obvious technical issues, current manufacturing practice is such that mechanical fasteners are still widely used in the construction of composite structures, especially when load transfer has to be achieved between composite and metallic components [11].

A typical example would be one in which the load from a composite wing skin is transferred into an aluminum metallic bracket through a bolted junction. When in service, each hole in the composite skin would be subjected to both tension and/or compression loading that may, under certain circumstances, promote damage initiation and damage progression.

Under tension, the composite joint may fail under three major modes: tear failure, bearing failure, and shear-out failure. Of these, the tear failure is unlikely to happen, because the fiber reinforcement is strongest in tension. The shear-out failure would happen if the fibers are predominant in the tension direction; shear-out failure can be counteracted through design by the addition of $45 \mathrm{deg}$ reinforcement. The bearing failure is more difficult to prevent, because it is a compression-type loading that has to be taken up by the polymeric matrix and by the fibers under compression. Bearing failure may occur through matrix crushing, or fiber microbuckling, or both.

Q10 Under compression, the composite joint may fail under three major modes: overall buckling of the component, local buckling of the region weaken by the hole, and fiber microbuckling at the areas of highest compression strength. The overall compression buckling can be prevented by proper component design. Local buckling and fiber microbuckling may be also prevented by design, but damage accumulation during cyclic loading would eventually weaken it.

\section{Impact Damage in Composite Structures}

Composite aerospace structures are prone to a particular type of damage that is not critical in metallic aerospace structures: i.e., lowvelocity impact damage. Such damage may occur during manufacturing or in service, due to, say, a hand tool being dropped onto a thin-wall composite part. When such an impact happens on a conventional metallic structural part, either the part is not damaged at all or, if it is damaged, then it shows clearly as an indent or scratch. In composite structures, a similar impact may damage the structure without leaving any visible marks on the surface (so-called barely visible damage). In this case, the impact result takes the form of delaminations in the composite layup. (A more drastic impact may also show spalling on the back side, while having no visible marks on the front side [12].)

Delamination due to barely visible impact damage may not have a large effect on the tension strength of the composite, but it can significantly diminish the composite compression strength (delaminated plies have a much weaker buckling resistance than the same plies solidly bonded together). Both component buckling strength and local buckling strength may be affected; when a fastening hole is present, this effect may be even worse. For this reason, manufacturing companies place a strong emphasis on testing the open-hole compression and compression after impact strengths of their composite structures. Detection of delaminations due to barely visible impact damage is a major emphasis in composite SHM research.

\section{E. Damage Detection in Composite Materials}

We have seen that the inherent macroscopic anisotropy and multimaterial architecture of the composite materials results in internal damage types that are significantly different from those encountered in isotropic metallic materials. Currently, one of the most commonly encountered damage types in composite materials is that caused by low-velocity impact; this damage susceptibility (which is not encountered in metallic structures) is mainly due to the low interlaminar strength of conventional composite layups. Significant degradation of the mechanical properties can easily occur as a result of low-velocity impact, even for barely visible damage. Different types of damage may be encountered in the impacted region, including matrix (resin) cracking, delaminations (interlaminar cracking), and broken fibers. In the future, as composite structures accumulate years of service, other damage types related to fatigue effects are also expected to become significant.

To date, much effort has been put into identifying reliable nondestructive evaluation (NDE) techniques for the detection, location, and characterization of composite materials damage, with special attention to subsurface delaminations due to manufacturing defects or to low-velocity impacts. Thermography, shearography, radiography, and ultrasonics are among the most commonly used NDE techniques [13]. These NDE techniques require stripping the aircraft and even removal of individual components; they employ bulky transducers, operate with point scanning, and, in general, are time-consuming, labor-intensive, and expensive. As a result, the current cost of composite structures inspection is very high: at least one order of magnitude greater than for metallic structures [14]. For composite structures to be economically viable and to realize their full design potential, it is essential that their operation and maintenance are conducted in a safe and economical manner, on par with that of existing metallic structures. For this reason, the development of new composite damage detection methods that can be applied rapidly and reliably to detect critical composite flaws is an ongoing concern. The permanently attached damage-sensing technologies developed under the SHM thrust are a promising research direction, because, when implemented, they would permit the interrogation at will of the composite structures and the reliable and credible detection of internal damage in order to increase flight safety and reduce operational costs. This technology, though promising, is still in its infancy, and many theoretical and experimental challenges still have to be resolved, as illustrated next.

\section{PWAS for Active SHM}

\section{A. PWAS Principles}

PWASs are the enabling technology for active SHM systems [15]. A PWAS couples the electrical and mechanical effects (mechanical strain $S_{i j}$, mechanical stress $T_{k l}$, electrical field $E_{k}$, and electrical displacement $D_{j}$ ) through the tensorial piezoelectric constitutive equations:

$$
S_{i j}=s_{i j k l}^{E} T_{k l}+d_{k i j} E_{k} \quad D_{j}=d_{j k l} T_{k l}+\varepsilon_{j k}^{T} E_{k}
$$

where $s_{i j k l}^{E}$ is the mechanical compliance of the material measured at zero electric field $(E=0), \varepsilon_{j k}^{T}$ is the dielectric permittivity measured at zero mechanical stress $(T=0)$, and $d_{k i j}$ represents the piezoelectric coupling effect. In practice, Voigt matrix notations are often used instead of tensor notation; for example, $d_{k i j}$ $(i, j, k=1,2,3)$ is replaced by $d_{q i}(q=1, \ldots, 6$ and $i=1,2,3)$. To generate guided waves in thin-wall structures, PWASs use the $d_{31}$ coupling between the in-plane strains $S_{1}$ and $S_{2}$ and transverse electric field $E_{3}$. When used to interrogate thin-wall structures, the PWASs are guided-wave transducers by coupling their in-plane motion with the guided-wave particle motion on the material surface. The in-plane PWAS motion is excited by the applied oscillatory voltage through the $d_{31}$ piezoelectric coupling. Optimum excitation and detection happens when the PWAS length is in certain ratios with the wavelength of the guided-wave modes. The PWASs action as ultrasonic transducers is fundamentally different from that of conventional ultrasonic transducers. Conventional ultrasonic transducers act through surface tapping: i.e., by applying vibration pressure to the structural surface. The PWAS transducers act through surface pinching, and are strain coupled with the structural surface. This allows the PWAS transducers to have a greater efficiency in transmitting and receiving ultrasonic surface and guided waves when compared with the conventional ultrasonic transducers. PWASs are lightweight and inexpensive and hence can be deployed in large numbers on the monitored structure. Just like conventional ultrasonic transducers, PWASs use the piezoelectric effect to generate and receive ultrasonic waves. However, PWASs are different from conventional ultrasonic transducers: 
1) PWASs are firmly coupled with the structure through an adhesive bonding, whereas conventional ultrasonic transducers are weakly coupled through gel, water, or air.

2) PWASs are nonresonant devices that can be tuned selectively into several guided-wave modes, whereas conventional ultrasonic transducers are resonant narrowband devices.

3) PWASs are inexpensive and can be deployed in large quantities on the structure, whereas conventional ultrasonic transducers are expensive and used one at a time.

As shown in Fig. 3, PWAS transducers can serve several purposes [15]: high-bandwidth strain sensors, high-bandwidth wave exciters and receivers, resonators, and embedded modal sensors with the electromechanical impedance method. By application types, PWAS transducers can be used for active sensing of far-field damage using pulse-echo, pitch-catch, and phased-array methods; active sensing of near-field damage using high-frequency electromechanical impedance method and thickness-gauge mode; and passive sensing of damage generating events through detection of low-velocity impacts and acoustic emission at the tip of advancing cracks.

By using Lamb waves in a thin-wall structure, one can detect structural anomaly: i.e., cracks, corrosions, delaminations, and other damage. Because of the physical, mechanical, and piezoelectric properties of PWAS transducers, they act as both transmitters and receivers of Lamb waves traveling through the structure. Upon excitation with an electric signal, the PWASs generate Lamb waves in a thin-wall structure. The generated Lamb waves travel through the structure and are reflected or diffracted by the structural boundaries, discontinuities, and damage. The reflected or diffracted waves arrive at the PWAS, where they are transformed into electric signals.

\section{B. Traveling-Wave Methods of Damage Detection with PWAS Transducers}

Figure 3a illustrates the pitch-catch method. An electric signal applied at the transmitter PWAS generates, through piezoelectric transduction, elastic waves that transverse the structure and are captured at the receiver PWAS. As long as the structural region between the transmitter and receiver is in pristine condition, the received signal will be consistently the same; if the structure becomes damaged, then the received signal will be modified. Comparison between the historically stored signals and the currently read signal will indicate when changes (e.g., damage) take place in the structure. The pitch-catch method may be applied to situations in which the damage is diffuse and/or distributed, such as corrosion in metals or degradation in composites. By extension of the pitch-catch method to several pitch-catch pairs in a network of PWASs (sparse array) placed around a structural region of interest, one achieves ultrasonic tomography. In such a network, all the PWASs are eventually paired through a round-robin process. The processing of all the collected data during the round-robin process yields an image of the monitored region indicating the damage area.

Figure $3 \mathrm{~b}$ illustrates the pulse-echo method. In this case, the same PWAS transducer acts as both transmitter and receiver. A tone-burst signal applied to the PWAS generates an elastic wave packet that travels through the structure and reflects at structural boundaries and at cracks and abrupt discontinuities. In a pristine structure, only boundary reflections are present, whereas in a damaged structure, reflections from cracks also appear. By comparing historical signals one can identify when new reflections appear, due to new boundaries such as cracks and abrupt discontinuities. This comparison may be facilitated by the differential signal method.

Figure $3 \mathrm{c}$ illustrates the use of PWAS transducers in thickness mode. The thickness mode is usually excited at much higher frequencies than the guided-wave modes discussed in the previous two paragraphs. For example, the thickness mode for a $0.2 \mathrm{~mm}$ PWAS is excited at around $12 \mathrm{MHz}$, whereas the guided-wave modes are excited at tens and hundreds of kilohertz. When operating in thickness mode, the PWAS transducer can act as a thickness gauge. In metallic structures, thickness-mode measurements allow the detection of damage that affects the structural thickness, e.g., corrosion, which can be detected from that side of the structure, which is outside of the corrosive environment. In composite structures, thickness-mode measurements may detect cracks that are parallel to the surface, such as delaminations. However, a limitation of the thickness-mode approach is that detection can only be made directly under the PWAS location, or in its proximity. In this respect, this method is rather localized, which may be sufficient for monitoring well-defined critical areas, but insufficient for large-area monitoring.

Figure $3 \mathrm{~d}$ illustrates the detection of impacts and acoustic emission $(\overline{\mathrm{AE}})$ events. In this case, the PWAS transducer is operating

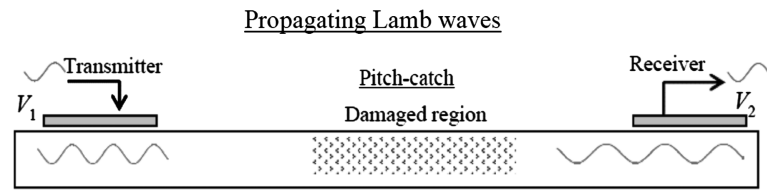

a)

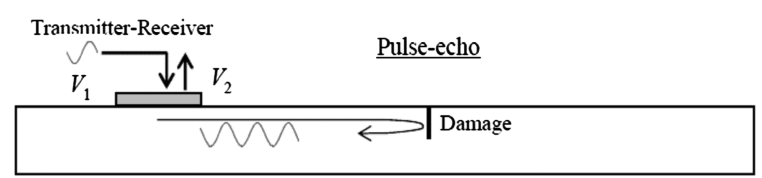

b)

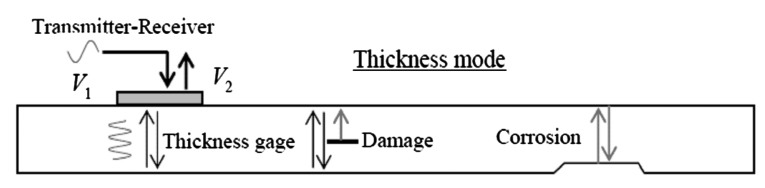

c)

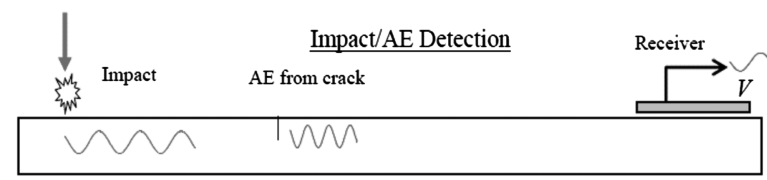

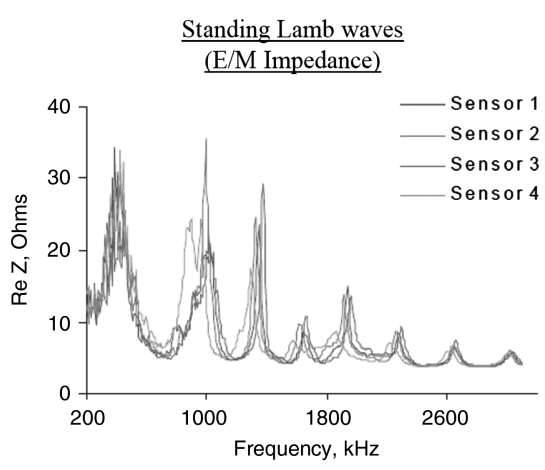

e)

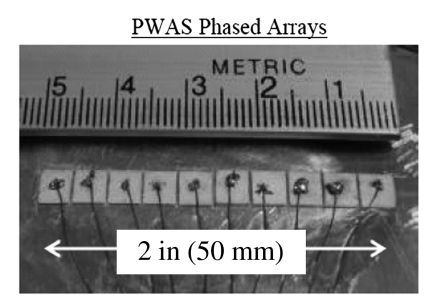

d)

Fig. 3 Use of a PWAS for damage detection with propagating and standing guided waves in thin-wall structures. 
as a passive receiver of the elastic waves generated by the impact or by the AE source. By placing several PWAS transducers in a network configuration around a given structural area, one can set up a listening system that would monitor if impact damage or AE events take place. Because the PWAS is self-energized through piezoelectric transduction, the listening system can stay in a low-energy dormant mode until a triggering by the PWAS wakes it up. The signals recorded by the PWAS network can be processed to yield the location and amplitude of the impact and/or AE event.

\section{Standing-Wave Methods of Damage Detection with PWAS Transducers}

When a structure is excited with sustained harmonic excitation of a given frequency, the waves traveling in the structure undergo multiple boundary reflections and settle down in a standing-wave pattern known as vibration. Structural vibration is characterized by resonance frequencies at which the structural response goes through peak values. The structural response measured over a frequency range including several resonance frequencies generates a vibration spectrum or frequency response function. When damage occurring in a structure induces changes in its dynamic properties, the vibration spectrum also changes. However, the conventional vibration analysis methods are not sensitive enough to detect small incipient damage; they can only measure structural dynamics up to several kilohertz, which is insufficient for the small wavelength needed to discover incipient damage. An alternative approach that is able to measure structural spectrum into the hundreds of kilohertz and low-megahertz range is offered by the electromechanical impedance method [15]. The electromechanical impedance method measures the electrical impedance $Z(\omega)$ of a PWAS transducer using a an impedance analyzer. The real part of the impedance $\operatorname{Re}(Z)$ reflects the mechanical behavior of the PWAS: i.e., its dynamic spectrum and its resonances. When the PWAS is attached to a structure, the real part of the impedance measured at the PWAS terminals reflects the dynamics of the structure on which the PWAS is attached: i.e., the structural dynamic spectrum and its resonances. Thus, a PWAS attached to a structure can be used as a structural-identification sensor that measures directly the structural response at very high frequencies. Figure $3 \mathrm{e}$ illustrates the electromechanical impedance spectrum measured in the megahertz range.

\section{Phased Arrays and the Embedded Ultrasonics Structural Radar}

A natural extension of the PWAS pulse-echo method is the development of a PWAS phased array (Fig. 3f) that is able to scan a large area from a single location. Phased arrays were first used in radar applications, because they allowed the replacement of the rotating radar dish with a fixed panel equipped with an array of transmitter-receivers that were energized with prearranged phase delays. When simultaneous signals are emitted from an array of transmitters, the constructive interference of the propagating waves creates a beam positioned broadside to the array. If prearranged phase delays are introduced in the firing of the signals of individual array elements, then the constructive interference beam can be steered to difference angular positions. Thus, an azimuth and elevation sweep can be achieved without mechanical rotation of the radar platform. The phased-array principle has gained wide use recently in ultrasonics, both for medical applications and for nondestructive evaluation, because ultrasonic phased arrays permit the sweeping of a large volume from a single location.

The PWAS phased arrays use the phase-array principles to create a interrogating beam of guided waves that travel in a thin-wall structure and can sweep a large area from a single location. The embedded ultrasonics structural radar (EUSR) methodology uses the signals collected by the PWAS phased array to recreate a virtual sweeping of the monitored structural area. The associated image represents the reconstruction of the complete area as if the interrogating beam was actually sweeping it. When no damage is present, the only echoes are those arriving from the natural boundaries of the interrogated area; if damage is present, its echo reflection is imaged on the EUSR screen indicating its location in $(R, \theta)$ or $(x, y)$ coordinates. Giurgiutiu et al.
[16] have used PWAS phased arrays to monitor crack growth during fatigue testing.

\section{Guided Waves in Composites}

The evaluation of structural integrity using Lamb-wave ultrasonics has long been acknowledged as a very promising technique. Several investigators $[17,18]$ have envisioned the inspection of large metallic plates from a single location using transducer arrays, where each element acts as both transmitter and receiver. Guided signals are generated at different angles around the transducer positions and the signal reflections from the boundaries are processed for damage detection. This configuration is very promising for isotropic material but might have some limitations for fibrous composite structures, due to the change in properties with fiber direction. In recent years, numerous investigations have explored Lamb-wave techniques for the detection of damage in composite laminates $[\underline{6}, 19,20]$. To take full advantage of Lamb-wave techniques for composite damage detection, one needs to first understand and model how guided waves propagate in composite structures, which are much more complicated than in isotropic metallic structures.

The guided waves propagating in composite structures are more difficult to model than those propagating in isotropic metallic structures because of the composite material is inherent anisotropy and multilayered, with each layer having a different orientation. For a plate made of one layer made of unidirectional fibers, the wave speed of the wave propagating in the material depends on the angle $\theta$ between fibers direction and wave-propagation direction. Hence, for each angle $\theta$, different dispersion curves will be derived. To obtain the dispersion curves in a plate made of more then one layer, for each layer in the plate, we must define a relation between displacements and stresses at the bottom surface and those at the top surface. Then, through the Snell low, the continuity of displacements, and the relation derived for each layer between stresses and displacements, we relate the stresses and the displacements at the bottom surface of the plate to those at the top surface of the plate. By imposing the stress-free boundary surfaces, we obtain the dispersion curves for the plate for a given propagation direction. Two solution approaches exist: the global matrix approach and the transfer matrix approach.

The global matrix method was proposed by Knopoff [21]. In the global matrix method, the equations for all the layers are considered simultaneously. There is no a priori assumption on the interdependence between the sets of equations for each interface. This process results in a system of $M=4(N-1)$ equations that has a narrowband $M \times M$ matrix. This technique is robust but slow to compute for many layers, because the matrix is rather large.

The transfer matrix (TM) approach [22-24] was use for layered composites by Nayfeh [25]. In the TM method, the equations for each layer are constructed separately and then linked together through the boundary conditions at the bottom and top surfaces of each layer. The process results in system of six equations for each layer.

Both methods share a common characteristic: a solution of the characteristic function does not strictly prove the existence of a modal solution, but only that the system matrix is singular. Furthermore, the calculation of the determinant for the modal solution needs the use of a good algorithm, because the aim of the problem is to find the zero of the determinant, whereas the matrix is frequently close to being singular.

Hereunder, we explain in some detail the procedure for the TM method. Figure 4 shows a composite plate made of $N$ layers; each layer is made of unidirectional fibers, and they are hence layers of orthotropic properties; however, the layer orientation varies from layer to layer [26]. Consider a wave propagating in the material and assume that the angle between the fiber direction and the direction of wave propagation for the $k$ th layer is $\theta_{k}$ (see Fig. 4). Define the global coordinate system $x_{1}, x_{2}, x_{3}$ such that $x_{1}$ is aligned with the direction of wave propagation. Also define a local coordinate system $x_{1}^{\prime}, x_{2}^{\prime}, x_{3}^{\prime}$ such that $x_{1}^{\prime}$ is parallel to the fiber direction (principal axes). The stiffness matrix of the $k$ th layer can be written as 


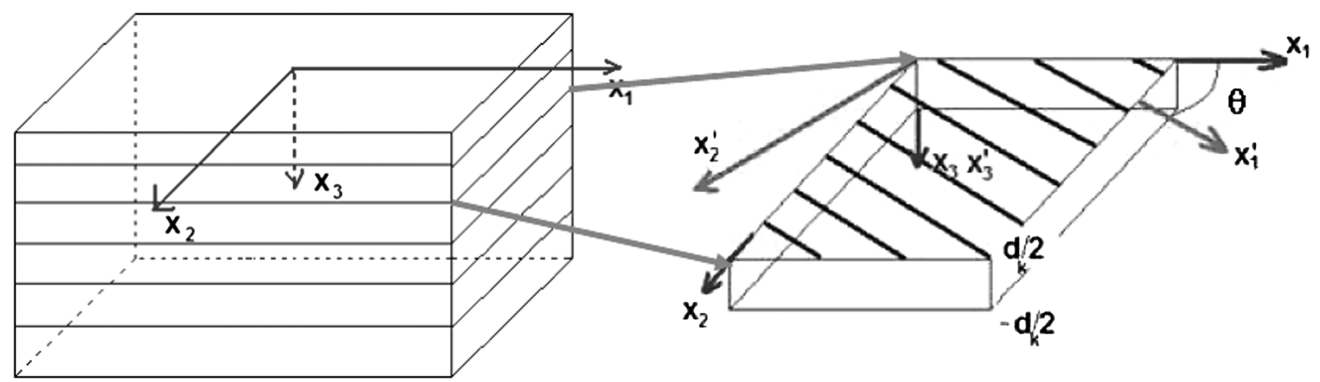

Fig. 4 Laminated composite plate illustrating the orientation of its $k$ th layer indicating the principal axes alignment with the layer unidirectional fibers.

$$
C=T_{1}^{-1} C^{\prime} T_{2}=\left[\begin{array}{cccccc}
C_{11} & C_{12} & C_{13} & 0 & 0 & C_{16} \\
C_{12} & C_{22} & C_{23} & 0 & 0 & C_{26} \\
C_{13} & C_{23} & C_{33} & 0 & 0 & C_{36} \\
0 & 0 & 0 & C_{44} & C_{45} & 0 \\
0 & 0 & 0 & C_{45} & C_{44} & 0 \\
C_{16} & C_{26} & C_{36} & 0 & 0 & C_{66}
\end{array}\right]
$$

where $C^{\prime}$ is the layer stiffness in local principal axes, $C$ is the stiffness matrix in global axes aligned with the direction of wave propagation, and $T_{1}$ and $T_{2}$ are the coordinate transformation matrices for stress and strain, respectively. Assume that the wave-propagation direction is parallel to the plane $x_{1} x_{2}$ and assume that the displacement solution can be written as

$$
\left(u_{1}, u_{2}, u_{3}\right)=\left(U_{1}, U_{2}, U_{3}\right) e^{i \xi\left(x_{1}+\alpha x_{3}-c t\right)}
$$

where $\xi$ is the wave number in the $x_{1}$ direction, $c=\omega / \xi$ is the phase velocity, $\omega$ is the angular frequency, $\alpha$ is an unknown parameter equal to the ratio between the wave numbers in the $x_{3}$ and $x_{1}$ directions, and $U_{i}$ is the displacement amplitude. The equation of motion in each composite layer can be solved to yield the expressions for the displacement field $\left(u_{1}, u_{2}, u_{3}\right)$ in terms of the layer properties: i.e.,

$$
\left\{\begin{array}{l}
\left(C_{11}+C_{55} \alpha^{2}-\rho c^{2}\right) U_{1}+\left(C_{16}+C_{45} \alpha^{2}\right) U_{2}+\left(C_{13}+C_{55}\right) \alpha U_{3}=0 \\
\left(C_{16}+C_{45} \alpha^{2}\right) U_{1}+\left(C_{66}+C_{44} \alpha^{2}-\rho c^{2}\right) U_{2}+\left(C_{45}+C_{36}\right) \alpha U_{3}=0 \\
\left(C_{13}+C_{55}\right) \alpha U_{1}+\left(C_{36}+C_{45}\right) \alpha U_{2}+\left(C_{55}+C_{33} \alpha^{2}-\rho c^{2}\right) U_{3}=0
\end{array}\right.
$$

If the material coordinate and the global coordinate systems coincide, the stiffness coefficients $C_{16}, C_{26}, C_{36}$, and $C_{45}$ are equal to zero; hence, the second equation in Eq. (4) becomes decoupled from the other two. This means that the SH wave is decoupled by the other two modes of propagation and the mathematical formulation is simpler. The system in Eq. (4) accepts nontrivial solution if the determinant is equal to zero. If we assume to know the velocity of the wave, the coefficients the characteristic equation gives three pairs of roots: i.e.,

$$
\alpha_{2}=-\alpha_{1}, \quad \alpha_{4}=-\alpha_{3}, \quad \alpha_{6}=-\alpha_{5}
$$

Since the determinant of Eq. (ㅁ) is equal to zero for any $\alpha_{q}$ ( $q=1,2, \ldots 6$ ), we find the solution to the system to be

$$
\left\{\begin{array}{l}
V_{q}=\frac{U_{2 q}}{U_{1 q}}=\frac{\left(c_{16}+c_{45} \alpha_{q}^{2}\right)\left(c_{13}+c_{55}\right)-\left(c_{11}+c_{55} \alpha_{q}^{2}-\rho v^{2}\right)\left(c_{45}+c_{36}\right)}{\left(c_{16}+c_{45} \alpha_{q}^{2}\right)\left(c_{45}+c_{36}\right)-\left(c_{66}+c_{44} \alpha_{q}^{2}-\rho v^{2}\right)\left(c_{13}+c_{55}\right)} \\
W_{q}=\frac{U_{3 q}}{U_{1 q}}=\frac{\left(c_{13}+c_{55}\right)\left(c_{16}+c_{45} \alpha_{q}^{2}\right) \alpha_{q}-\left(c_{11}+c_{55} \alpha_{q}^{2}-\rho v^{2}\right)\left(c_{36}+c_{45}\right) \alpha_{q}}{\left(c_{13}+c_{55}\right)\left(c_{36}+c_{45}\right) \alpha_{q}^{2}-\left(c_{55}+c_{33} \alpha_{q}^{2}-\rho v^{2}\right)\left(c_{16}+c_{45} \alpha_{q}^{2}\right)}
\end{array}\right.
$$

With the use of Eq. (), solution (3) can be written as

$$
\left(u_{1}, u_{2}, u_{3}\right)=\sum_{q=1}^{6}\left(1, V_{q}, W_{q}\right) U_{1 q} e^{i \xi\left(x_{1}+\alpha_{q} x_{3}-v t\right)}
$$

To obtain the stress relation in terms of the displacement amplitude $U_{i}$, we substitute solution (7) in the generic stress-displacement relation. Since we want to relate the stresses on the top surface with those on the bottom, we consider only the stresses on the plane normal to direction 3 : i.e.,

$$
\left(\sigma_{33}^{*}, \sigma_{13}^{*}, \sigma_{23}^{*}\right)=\sum_{q=1}^{6}\left(D_{1 q}, D_{2 q}, D_{3 q}\right) U_{1 q} e^{i \xi\left(x_{1}+\alpha_{q} x_{3}-v t\right)}
$$

where $\sigma^{*}=\sigma / i \xi$ and

$$
\begin{aligned}
& D_{1 q}=c_{13}+\alpha_{q} c_{33} W_{q}+c_{36} V_{q} \\
& D_{2 q}=\alpha_{q} c_{45} V_{q}+c_{55}\left(\alpha_{q}+W_{q}\right) \\
& D_{3 q}=\alpha_{q} c_{44} V_{q}+c_{45}\left(\alpha_{q}+W_{q}\right)
\end{aligned}
$$

The relation for the wave propagation in a layer is given by the combination of the displacement and stress relations: i.e.,

$$
\begin{aligned}
{\left[\begin{array}{l}
u_{1} \\
u_{2} \\
u_{3} \\
\sigma_{33}^{*} \\
\sigma_{13}^{*} \\
\sigma_{23}^{*}
\end{array}\right]=\left[\begin{array}{cccccc}
1 & 1 & 1 & 1 & 1 & 1 \\
V_{1} & V_{1} & V_{3} & V_{3} & V_{5} & V_{5} \\
W_{1} & -W_{1} & W_{3} & -W_{3} & W_{5} & -W_{5} \\
D_{11} & D_{11} & D_{13} & D_{13} & D_{15} & D_{15} \\
D_{21} & -D_{21} & D_{23} & -D_{23} & D_{25} & -D_{25} \\
D_{31} & -D_{31} & D_{31} & -D_{31} & D_{35} & -D_{35}
\end{array}\right] } \\
\times\left[\begin{array}{c}
U_{11} e^{i \xi \alpha_{1} x_{3}} \\
U_{11} e^{-i \xi \alpha_{1} x_{3}} \\
U_{13} e^{i \xi \alpha_{3} x_{3}} \\
U_{13} e^{-i \xi \alpha_{3} x_{3}} \\
U_{15} e^{i \xi \alpha_{5} x_{3}} \\
U_{15} e^{-i \xi \alpha_{5} x_{3}}
\end{array}\right] e^{i \xi\left(x_{1}-v t\right)}
\end{aligned}
$$

After the wave propagation inside each layer is setup in terms of layer properties and boundary conditions at the top and bottom contact surfaces with the other layers, the next step is to solve for wave propagation in the complete composite laminate. This is achieved by linking together the composite layers by imposing equilibrium and compatibility conditions at the interfaces: i.e., matching stresses and displacements.

The tractions and strains at the bottom of one layer are described in terms of those of the top of the layer through a transfer matrix, which depends on the properties and orientation of that specific layer: i.e.,

$$
\left\{\begin{array}{l}
\left\{u_{k}^{+}\right\} \\
\left\{\sigma_{k}^{+}\right\}
\end{array}\right\}=\left[A_{k}\right]\left\{\begin{array}{l}
\left\{u_{k}^{-}\right\} \\
\left\{\sigma_{k}^{-}\right\}
\end{array}\right\}
$$

where the matrix $A_{k}$ is the layer-transfer matrix for the $k$ th layer. To define matrix $A_{k}$, call the $6 \times 6$ matrix of Eq. (10) $X$, the vector of the $U_{1 i}$ elements $U$, and the diagonal matrix whose elements are $e^{i \xi \alpha_{i} x_{3}}$ $D$. Hence,

$$
A_{k}=X_{k} D_{k} X_{k}^{-1}
$$

The transfer between two consecutive layers is achieved by imposing displacements continuity force equilibrium at the interfaces between consecutive layers. Thus, boundary conditions at the top of the composite plate (e.g., wave excitation) are propagated from layer to layer to the bottom of the plate through the sequential layer-to-layer 
transfer. In the end, we relate the displacements and stresses of the top of the layered plate to those at its bottom through the overall transfer matrix $A$, given by

$$
A=\prod_{k=1}^{N} A_{k}
$$

Thus, a small linear system of equation is setup in which the boundary conditions at the top of the plate are related to the boundary conditions at bottom of the plate. To find the dispersion curves and mode shapes, one assumes traction-free conditions at both top and bottom surfaces and solves the resulting homogenous system in terms of unknown top and bottom displacements. The resulting eigenvalue problem yields eigenwave numbers and the associate eigendisplacements at the tops and bottoms of the plates. The eigenwave numbers yield the phase velocities at the assumed frequency; the dispersion curves are then obtained by repeating the process over a frequency range. The eigendisplacements are propagated through the matrix transfer process through all the layers in order to determine the thickness-mode shape of that particular guided-wave mode. Though simple in formulation, the TM method suffers from numerical instability, because error accumulates in the layer transfers. To address the numerical instabilities, Rokhlin and Wang [27] address this numerical instability issue by introducing the layer stiffness matrix and using an efficient recursive algorithm to calculate the global stiffness matrix for the complete laminate. The layer stiffness matrix relates the stresses at the top and bottom of the layer with the corresponding displacements. The terms in the matrix have only exponentially decaying terms, and hence the transfer process becomes more stable.

\section{A. Dispersion Curves for Composite Structures}

Q17 We coded the TM approach following Nayfeh [25]. Figure $\underline{6}$ shows the dispersion curves derived for a unidirectional composite plate made of one layer of $65 \%$ graphite $35 \%$ epoxy [26]. These dispersion curves depend on the propagation direction, $\overline{\theta ;}$ the cases $\theta=0,18,36$, and $90 \mathrm{deg}$ are presented in Fig. 6 . It is apparent that for $\theta=0$ deg (i.e., wave propagating along the fiber direction) and for $\theta=90^{\circ}$ (i.e., waves propagating transversely to the fiber direction), the dispersion curves are clearly decoupled into quasi-antisymmetric horizontal $\left(\mathrm{SH}_{0}, \mathrm{SH}_{1}, \mathrm{SH}_{2}, \ldots\right)$ wave modes. However, this is not the case for the offaxis directions $\theta=18,36 \mathrm{deg}$, in which case the three mode types are strongly coupled. The wave velocity is higher when the wave propagates along the fiber direction. As the angle of the wave-propagation direction increases, the phase velocity decreases till reaching a minimum in the direction perpendicular to the fiber. This is due to the fact that along the fiber the material stiffness is

greater than in all the other directions and it decreases, whereas $\theta$ increases.

Next, we analyzed various composite layups. Figure $\underline{5}$ shows the dispersion curves for a $\left[(0 / 45 / 90 /-45)_{2 s}\right]$ layup. Figure $\underline{5}$ a shows the dispersion curves over a large $\xi d$ range: a complicated pattern of tightly coupled composite-plate wave modes is present. Figure $\underline{5 b}$ presents a zoom-in to low- $\xi d$ values, in which case only the $\mathrm{A}_{0}, \overline{\mathrm{S}_{0}}$, and $\mathrm{SH}_{0}$ modes are present.

\section{B. Group Velocity in Composite Structures}

In isotropic metallic plates, the calculation of group velocity $c_{g}$ (also known as energy velocity $c_{E}$ ) is rather straightforward, since it is done by differentiation of frequency with respect to wave number: i.e., $c_{g}=\partial \omega / \partial \xi$. In anisotropic fibrous composite, the calculation of group velocity is rather more complicated and it has to use the slowness curve (Fig. 7). The phase slowness is the inverse of the phase velocity; hence, the phase slowness curve shows how the relative arrival time of a plane wave propagating in an anisotropic plate varies with the direction of wave propagation. Assume we want to find the group velocity $c_{E}$ for a wave propagating along direction $\theta$ with respect to the fiber direction.

By definition, the group velocity vector is perpendicular to the phase slowness curve. Consider the slowness curve of Fig. $\underline{7}$ and a point of interest $P$ corresponding to the propagation direction $\theta$. By drawing the normal to the slowness curve at point $P$ we determine the direction of the group velocity vector $c_{E}$; this direction is oriented along angle $\psi$. By elementary geometry applied to Fig. 7, the group velocity magnitude is given by $c_{E}=c / \cos \phi$, where $\phi=\psi-\theta$. Knowing the magnitude of $c_{E}$ and the angle $\psi$ for each propagation direction, one can construct the wave surface.

Figure 8a shows the slowness curve for a 65/35 graphite/epoxy unidirectional plate. The slowness curve is derived from the inverse of the phase velocity for every possible angle wave propagation at a given frequency-thickness product. Two frequency-thickness product values are plotted: $f d=400$ and $1700 \mathrm{kHz} \cdot \mathrm{mm}$; the slowness curves are different for each frequency-thickness product. Figure $\underline{8 b}$ shows the corresponding wave-front contours. It is apparent that the wave propagation is faster along the fiber direction than across the fiber direction. This observation is consistent with the fact that the fibrous composite is much stiffer along the fibers than across the fibers. The fact that the wave-front contour varies with the frequency-thickness product is also apparent in Fig. $\underline{8 b}$.

\section{Tuned Guided Waves in Composite Structures}

The tuning between PWAS transducers and guides waves in isotropic metallic plates is relatively well understood and modeled. The gist of the concept is that manipulation of PWAS size and

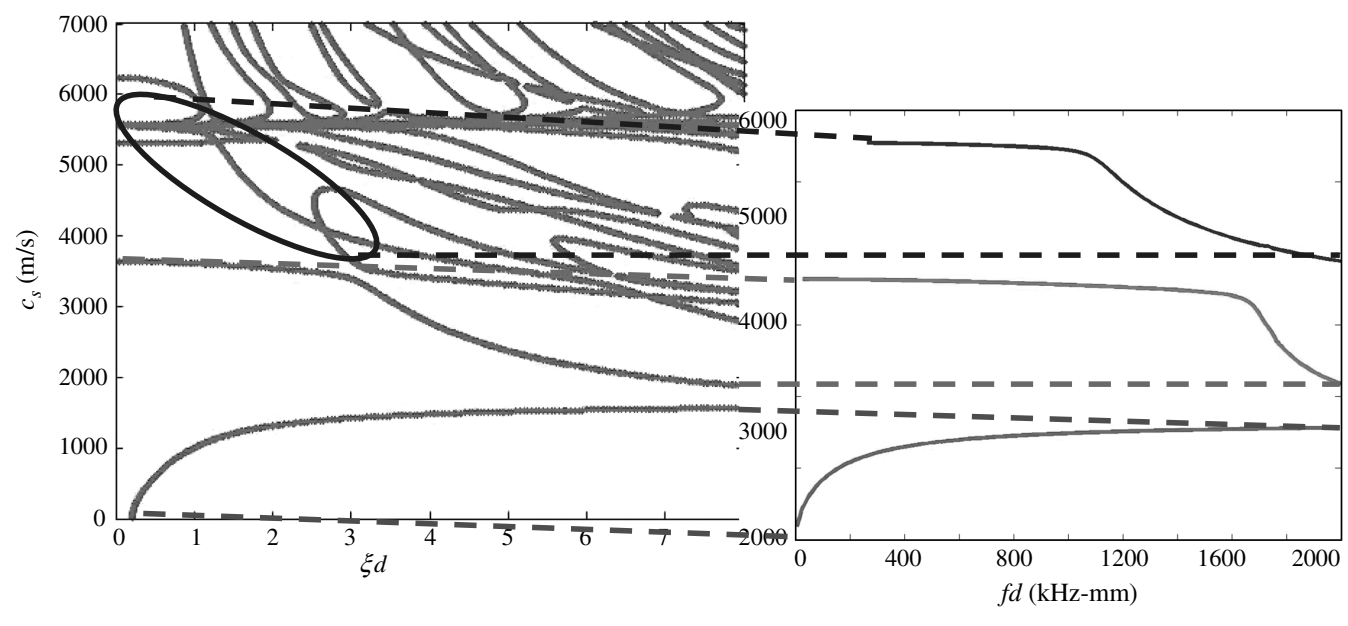

a)

b)

Fig. 5 Dispersion curves for a quasi-isotropic plate $\left[(0 / 45 / 90 /-45)_{2 s}\right]$ wave propagating in the 0 direction: a) output from the program and b) elaboration of $\mathrm{S}_{0}, \mathrm{SH}$, and $\mathrm{A}_{0}$ modes. 


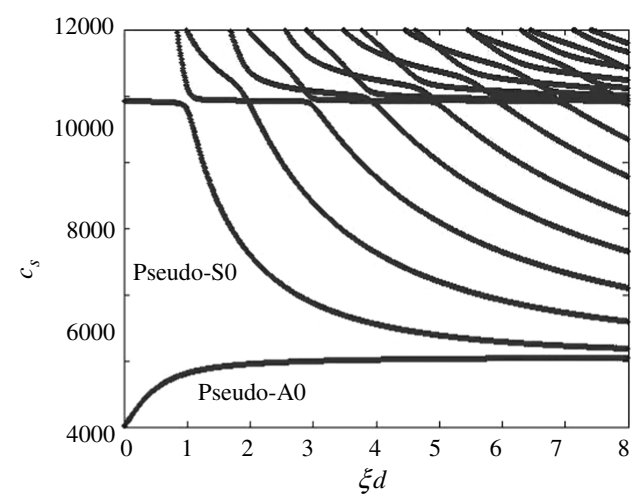

a)

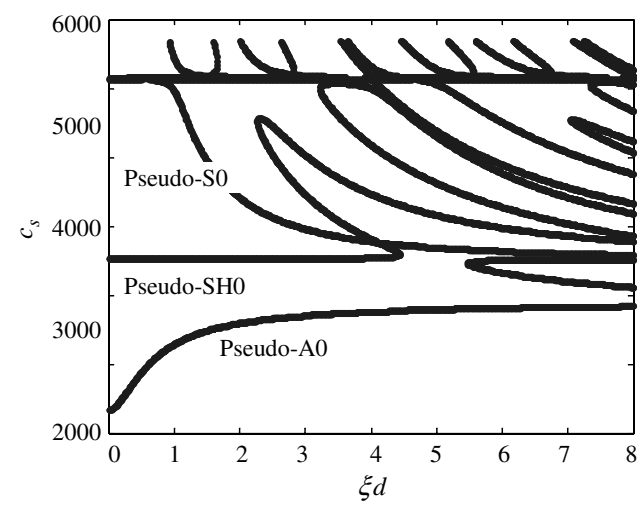

c)

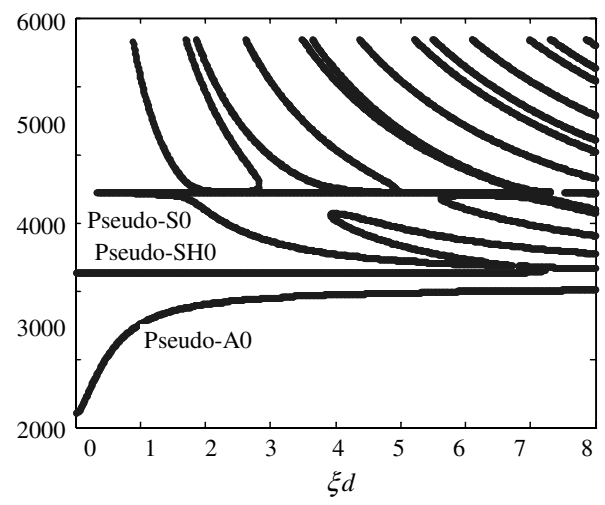

b)

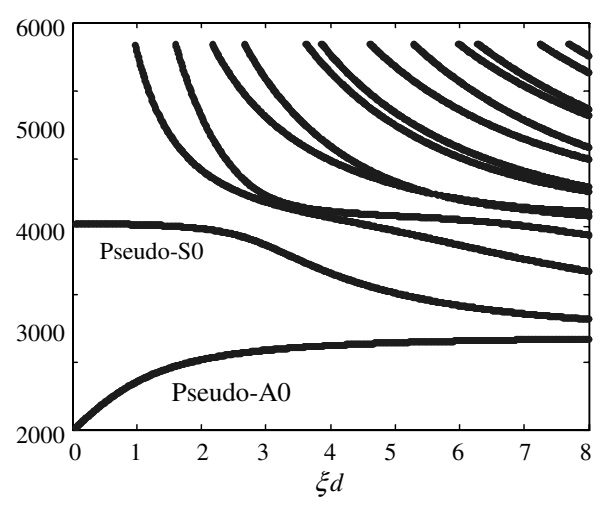

d)

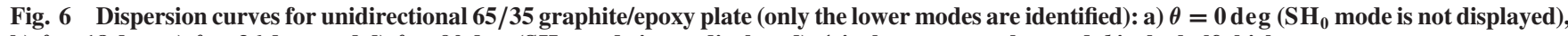
b) $\theta=18 \mathrm{deg}$, c) $\theta=36 \mathrm{deg}$, and d) $\theta=90 \mathrm{deg}\left(\mathrm{SH}_{0}\right.$ mode is not displayed); $\xi$ is the wave number and $d$ is the half-thickness.

frequency allow for selective preferential excitation of certain guided-wave modes and the rejection of other guided-wave modes, as needed by the particular SHM application under consideration. A similar tuning effect is also possible in anisotropic composite plates, but the analysis is more complicated, due to the anisotropic wavepropagation characteristics inherent in composite materials [26].

The analysis is performed in two steps:

1) Perform the free-response analysis and solve the homogenous problem to determine the dispersion curves and wave modes over the frequency domain of interest.

2) Perform the force-response analysis in which the PWAS transducer is used to excite guided-wave modes in the composite plate (Fig. 9). The analysis uses the normal mode expansion, since, at any arbitrary frequency, several wave modes can be excited. Some details of our approach are given below; further details are available in [26].

The starting point is the complex reciprocity relation under the assumption of time harmonic waves: i.e.,

$$
\begin{aligned}
& \frac{\delta}{\delta y}\left(-\tilde{\mathbf{v}}_{2} \cdot \mathbf{T}_{1}-\mathbf{v}_{1} \cdot \tilde{\mathbf{T}}_{2}\right) \cdot \overline{\mathbf{y}}+\frac{\delta}{\delta x}\left(-\tilde{\mathbf{v}}_{2} \cdot \mathbf{T}_{1}-\mathbf{v}_{1} \cdot \tilde{\mathbf{T}}_{2}\right) \cdot \overline{\mathbf{x}} \\
& \quad=\tilde{\mathbf{v}}_{2} \mathbf{F}_{1}+\mathbf{v}_{1} \tilde{\mathbf{F}}_{2}
\end{aligned}
$$

where $\mathbf{v}$ is the particle velocity vector, $\mathbf{T}$ is the stress tensor, $\mathbf{F}$ is the applied external force, 1 and 2 are two different solutions due to two different external forces, and the tilde means the complex conjugate.

Consider the case of a PWAS bonded on the top surface of a composite plate. In this case, the wave guides can be excited at the acoustic boundaries by traction forces only, $\mathbf{T} \cdot \overline{\mathbf{y}}$. We assume that the excited field (solution 1) can be represented by mode expansion: i.e.,

$$
\begin{aligned}
\mathbf{v}_{1} & =\mathbf{v}_{1}(x, y)=\sum_{n} a_{n}(x) \mathbf{v}_{n}(y) \\
\mathbf{T}_{1}=\mathbf{T}_{1}(x, y) & =\sum_{n} a_{n}(x) \mathbf{T}_{n}(y)
\end{aligned}
$$

where $a_{m}(x)$ are the $x$-dependent modal participation factors that depend on the mode under consideration and the excitation used to generate the field. The modal participation factors are the same for the all the acoustic fields. The $y$-dependent terms, $\mathbf{v}_{n}(y)$ and $\mathbf{T}_{n}(y)$, are assumed to be known and depend only on the mode considered. We assume also that solution 2 is of the type

$$
\begin{aligned}
\mathbf{v}_{2} & =\mathbf{v}_{2}(x, y)=\mathbf{v}_{n}(y) e^{-i \xi_{n} x} \\
\mathbf{T}_{2}=\mathbf{T}_{2}(x, y) & =\mathbf{T}_{n}(y) e^{-i \xi_{n} x} \quad \text { with } \quad \mathbf{F}_{2}=0
\end{aligned}
$$

Integrating Eq. (14) with respect to $y$ and through the use of Eqs. (15) and (16) we obtain

$$
\left.\left(-\tilde{\mathbf{v}}_{n} \cdot \mathbf{T}_{1}-\mathbf{v}_{1} \cdot \tilde{\mathbf{T}}_{n}\right) \cdot \overline{\mathbf{y}}\right|_{-d} ^{d} e^{i \tilde{\xi}_{n} x}+\frac{\delta}{\delta x}\left(e^{i \tilde{\xi}_{n} x} \sum_{m} a_{m}(x) P_{n m}\right)=0
$$

where $P_{n m}$ is the average power flow of the $n$th guided mode: i.e.,

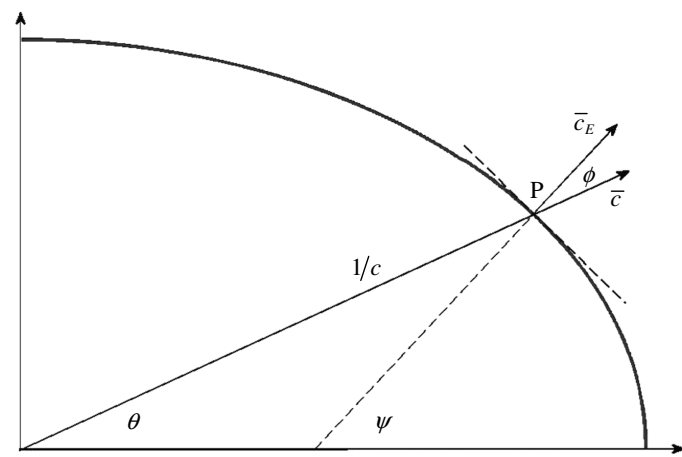

Fig. 7 Slowness curve and notation. 


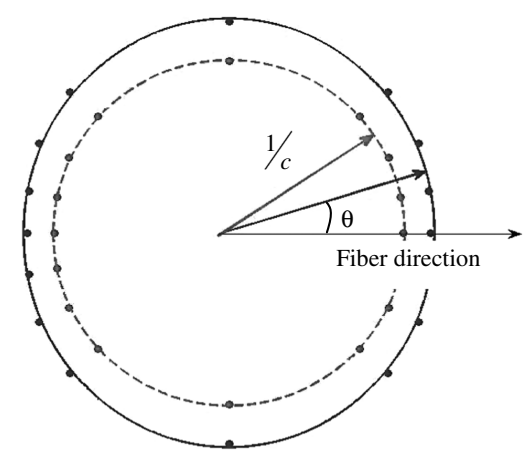

a)

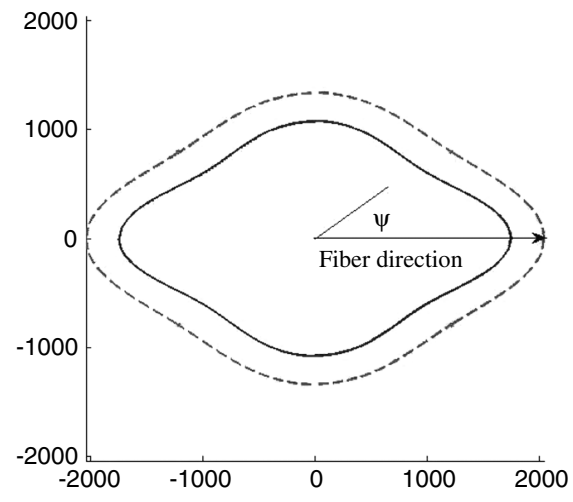

b)

Fig. 8 Directional dependence of wave propagation in unidirectional $65 \%$-graphite $/ 35 \%$-epoxy plate at $f d=400 \mathrm{kHz} \cdot \mathrm{mm}(\mathrm{solid}$ line) and $f d=$ $1700 \mathrm{kHz} \cdot \mathrm{mm}$ (dashed line): a) slowness curve (plotted values are $10^{4} 1 / \mathrm{c}$ ) and b) wave front.

$$
P_{n m}=\frac{1}{4} \int_{-d}^{d}\left(-\tilde{\mathbf{v}}_{n} \cdot \mathbf{T}_{m}-\mathbf{v}_{m} \cdot \tilde{\mathbf{T}}_{n}\right) \cdot \overline{\mathbf{x}} \mathrm{d} y
$$

According to the orthogonality relation of the wave modes [28], the summation in Eq. (17) has only one nonzero term. Considering the propagating mode $\bar{n}\left(\xi_{n}\right.$ real), Eq. (17) can be written as

$$
4 P_{n n}\left(\frac{\delta}{\delta x}+i \xi_{n}\right) a_{n}(x)=\left.\left(\tilde{\mathbf{v}}_{n} \cdot \mathbf{T}+\mathbf{v} \cdot \tilde{\mathbf{T}}_{n}\right) \cdot \overline{\mathbf{y}}\right|_{-d} ^{d}
$$

where

$$
\begin{aligned}
P_{n n} & =\operatorname{Re}\left[\frac{1}{4} \int_{-d}^{d}\left(-\tilde{\mathbf{v}}_{n} \cdot \mathbf{T}_{n}-\mathbf{v}_{n} \cdot \tilde{\mathbf{T}}_{n}\right) \cdot \overline{\mathbf{x}} \mathrm{d} y\right] \\
& =\operatorname{Re}\left[-\frac{1}{2} \int_{-d}^{d} \tilde{\mathbf{v}}_{n} \cdot \mathbf{T}_{n} \cdot \overline{\mathbf{x}} \mathrm{d} y\right]
\end{aligned}
$$

Assume that the anisotropic plate is loaded over a finite portion in the $y$ direction on the top surface by an infinite width traction force in the $x$ direction:

$$
\mathbf{T} \cdot \overline{\mathbf{y}}=\mathbf{t}(x) e^{i \omega t}=\left[t_{y}(x) \overline{\mathbf{y}}+t_{x}(x) \overline{\mathbf{x}}\right] e^{i \omega t}
$$

With the use of the load defined in Eq. (21) and noticing that the second term on the left-hand side is zero, because we assumed traction-free boundary, Eq. (19) becomes

$$
4 P_{n n}\left(\frac{\delta}{\delta x}+i \xi_{n}\right) a_{n}(x)=\tilde{\mathbf{v}}_{n}(d) \cdot \mathbf{t}(x)
$$

Q20 This is a first-order ordinary differential equation that governs the amplitudes of the general modes. Its solution is

$$
a_{n}(x)=\frac{e^{-i \xi_{n} x}}{4 P_{n n}} \tilde{\mathbf{v}}_{n}(d) \cdot \int_{c}^{x} e^{i x_{n} \eta} \mathbf{t}(\eta) \mathrm{d} \eta
$$

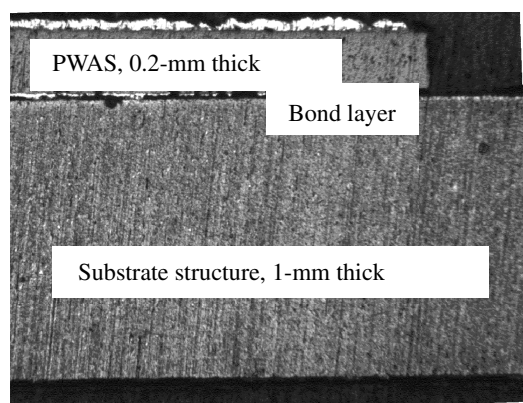

a)

Fig. 9 Model of bond-layer interaction between PWAS and composite structure.

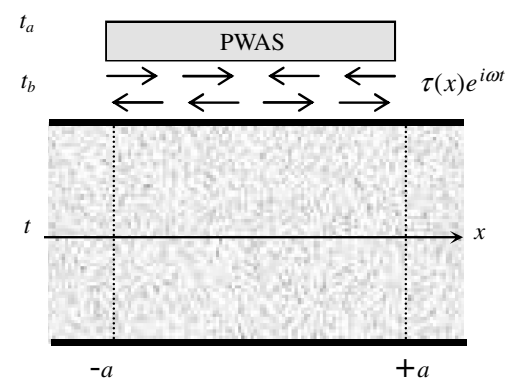

b) where $c$ is a constant used to satisfy the boundary conditions. Consider a PWAS of length $2 a$ bonded on the top surface of the composite plate. The external tractions $\mathbf{t}$ are nonzero only in the interval $-a \leq x \leq a$. We can write the forward-wave solution as

$$
a_{n}^{+}(x)=\frac{\tilde{\mathbf{v}}_{n}(d)}{4 P_{n n}} \cdot e^{-i \xi_{n} x} \int_{-a}^{a} e^{i \xi_{n} \bar{x}} \mathbf{t}(\bar{x}) \mathrm{d} \bar{x} \quad \text { for } x>a
$$

The strain (and hence the tuning curves) on the top surface of the plate is given by

$$
\varepsilon_{x}=\frac{\partial}{\partial x} \sum_{n} a_{n}(x) v_{x}(y) \int e^{i \omega t} \mathrm{~d} t=\frac{1}{\omega} \sum_{n} \xi_{n} a_{n}(x) v_{x}(y) e^{i \omega t}
$$

or in expanded form using Eq. (23):

$$
\varepsilon_{x}=\frac{\tilde{\mathbf{v}}_{n}(d)}{4 \omega P_{n n}} v_{x}(y) e^{i \omega t} \sum_{n} \xi_{n} e^{-i \xi_{n} x} \int_{-a}^{a} e^{i \xi_{n} \bar{x}} \mathbf{t}(\bar{x}) \mathrm{d} \bar{x} \quad \text { for } x>a
$$

This represents the tuning expression of the strain in the composite plate excited by the PWAS. This derivation is formally equal to the case of an isotropic plate. The number of modes present depends on the material properties of the composite plate. For the case of a composite plate made of one layer of unidirectional fibers, the PWAS will excite only Lamb modes (symmetric and antisymmetric) if we consider propagation along the fibers or transverse to the fibers. In all other cases, three waves will be present.

The main difficult in solving Eq. (26) lies in the derivation of the average power flow. The average power flow is given by the integral over the plate thickness of the velocity by the stress, and it must be performed numerically. Consider the $n$th wave mode propagating in the $k$ th layer of the composite plate (for simplicity of notation, we drop the subscript $n$ ), the integrand of Eq. (20) is given by

$$
(\tilde{\mathbf{v}} \cdot \mathbf{T}) \cdot \hat{\mathbf{x}}=\tilde{v}_{1} T_{1}+\tilde{v}_{2} T_{6}+\tilde{v}_{3} T_{4}
$$




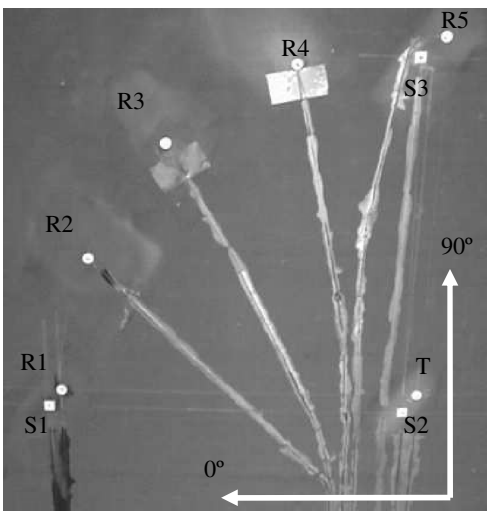

Fig. 10 Experiment setup measuring directional wave speeds in a [(0/45/90/ - 45)2 $]_{S}$ plate 1240 by $1240 \mathrm{~mm}$ with $2.25 \mathrm{~mm}$ thickness. The plate was laminated from T300/5208 unidirectional tape.

where the velocities are derived from the particle displacement solution (7): i.e.,

$$
\left(v_{1}, v_{2}, v_{3}\right)=-i \xi v \sum_{q=1}^{6}\left(1, V_{q}, W_{q}\right) U_{1 q} e^{i \xi\left(x_{1}+\alpha_{q} x_{3}-v t\right)}
$$

and the stresses are defined as

$$
\left\{\begin{array}{l}
T_{1} \\
T_{4} \\
T_{6}
\end{array}\right\}=i \xi \sum_{q}\left\{\begin{array}{c}
c_{11}+\alpha_{q} c_{13} W_{q}+c_{16} V_{q} \\
\alpha_{q} c_{44} V_{q}+c_{45}\left(\alpha_{q}+W_{q}\right) \\
c_{16}+\alpha_{q} c_{36} W_{q}+c_{66} V_{q}
\end{array}\right\} U_{1 q} e^{i \xi\left(x_{1}+\alpha_{q} x_{3}-v t\right)}
$$

Once the dispersion curves are known, the stress and the velocity in each layer for each mode are known, and hence the average power flow can be computed. It is to be emphasized that the derivation of the tuning curves presented here does not depend on the particular method used to derive the dispersion curves.

The integral in Eq. (26) depends on the assumption made on the bond layer between PWAS and structure. For the composite plate, we assume that the thickness of the bond layer is approaching zero; i.e., we assume ideal bond conditions.

In the case of ideal bonding solution, the shear stress in the bonding layer is concentrated at the ends of the PWAS tips. We can use the pin-force model to represent the load transferred form the PWAS to the structure: i.e.,

$$
\mathbf{t}(x, d)= \begin{cases}a \tau_{0}[\delta(x-a)-\delta(x+a)] \overline{\mathbf{x}} & \text { if }|x| \leq a \\ 0 & \text { if }|x|>a\end{cases}
$$

Substituting Eq. (30) into Eq. (26), after integration we obtain

$$
\varepsilon_{x}=i a \tau_{0} \frac{\tilde{\mathbf{v}}_{n}(d)}{2 \omega P_{n n}} v_{x}(y) e^{i \omega t} \sum_{n} \xi_{n} e^{-i \xi_{n} x} \sin \xi_{n} a
$$

where $a \tau_{0}$ is a constant that depends on the excitation, and $\tilde{v}_{n x}(d) / 4 P_{n n}$ is the excitability function of mode $n$ (depends on the mode excited and not on the source used for excitation).

It is apparent from Eq. (31) that as the wave number $\xi_{n}$ varies, the function $\sin \xi_{n} a$ goes from maxima and minima in the $(-1,+1)$ interval, and hence the response of the $n$th mode goes through peaks and valleys. The wave number $\xi_{n}$ varies with frequency $\omega$ and phase velocities $c_{n}$ : i.e., $\xi_{n}=\omega / c_{n}$. Since the variation of phase velocity with frequency is different from mode to mode, it is apparent that the peaks and valleys of one mode will not coincide with those of the other modes. Hence, one can find frequencies at which certain modes are rejected (valleys in their response curve), and other modes are tuned in (peaks in their response curves). It is also apparent that because guided-wave propagation in composite plates is directionally dependent, the tuning between the PWAS transducer and the guided waves in the composite plate will also depend on the propagation direction.

To verify these theoretical predictions we performed experiments on a composite plate with PWAS receivers installed at various directions from the PWAS transmitter. A $1240 \times 1240 \mathrm{~mm}$ quasiisotropic plate with a $[(0 / 45 / 90 /-45) 2]_{S}$ layup from T300/5208 carbon-fiber unidirectional tape was used. The plate had an overall thickness of $2.25 \mathrm{~mm}$. Figure 10 shows the central part of the composite plate, where $7 \mathrm{~mm}$ round PWAS transducers $(0.2-\mathrm{mm}$ thick, American Piezo Ceramics APC-850) were installed. The PWAS denoted with the letter $\mathrm{T}$ was the transmitter, and those denoted with $\mathrm{R}$ were the receivers (R1-R5). The distance between the receivers and the transmitter was $250 \mathrm{~mm}$. The step angle between sequential receivers was $\Delta \theta=22.5 \mathrm{deg}$. In addition, a pair of $7 \mathrm{~mm}$ square PWASs were placed along the fiber direction, with $\mathrm{S}_{1}$ being the transmitter and $S_{2}$ the receiver.

Smoothed three-count tone-burst excitation signals were used. The signal frequency was swept from 15 to $700 \mathrm{kHz}$ in steps of $15 \mathrm{kHz}$. At each frequency, the wave amplitude and the time of flight for all the waves present were collected. Since carbon-fiber is electrically conductive, the composite plate could be used as ground and only a single excitation wire had to be cabled to each PWAS transducer. We found that the ground quality affects the signal strength; to obtain a strong signal, a good electrical ground was achieved through bonding a sheet of copper on the composite surface. In this way the signal was strong and consistent during the experiments.

Three guided-wave modes were detected: quasi- $\mathrm{S}_{0}$, quasi- $\mathrm{A}_{0}$, and quasi- $\mathrm{SH}_{0}$. The identification of these three guided-wave modes was done using wave-packet group velocity compared with theoretical predictions. Figure 11a shows the experimentally measured signal amplitudes for the three guided waves for the $S_{1}-S_{2}$ transducer pair

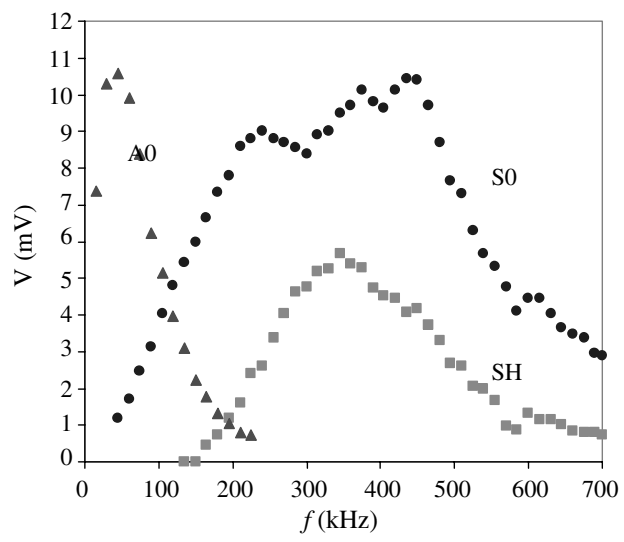

a)

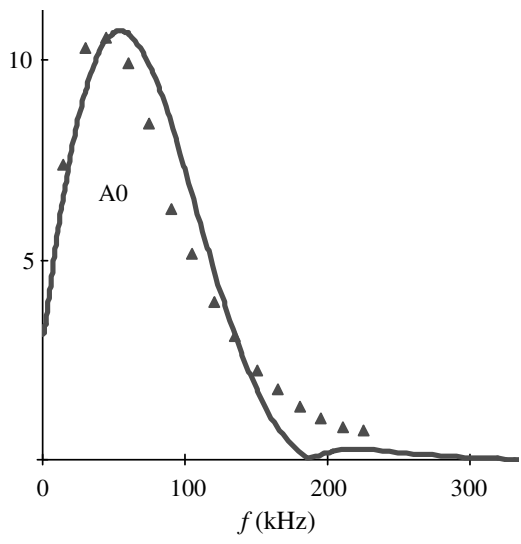

b)

Fig. 11 Tuning of PWAS guided waves in composites: a) quasi- $\mathrm{A}_{0}$ mode, quasi- $\mathrm{S}_{0}$ mode, and quasi-SH $\mathrm{S}_{0}$ mode for $\mathrm{PWASs}_{1}-\mathrm{S}_{2}$ and b) comparison of theoretical prediction (solid line) vs experimental values for $\mathbf{A}_{0}$ mode. 
propagating along the 0 deg direction. The quasi- $\mathrm{A}_{0}$ reaches a peak response at around $50 \mathrm{kHz}$ and then decreases. In fact, the $\mathrm{A}_{0}$ mode disappears as soon as the quasi- $\mathrm{SH}_{0}$ wave appears. The quasi- $\mathrm{S}_{0}$ mode reaches a peak at $450 \mathrm{kHz}$ and then decreases. The quasi- $\mathrm{SH}_{0}$ mode reaches a peak response at around $325 \mathrm{kHz}$. Figure $11 \mathrm{~b}$ shows a preliminary comparison between theoretical prediction derived through Eq. (31) and experimental values for the $\mathrm{A}_{0}$ mode propagating along the 0 deg direction; The vertical scale is nondimensional, since our focus has been on identifying the salient frequencies. Tuning (maximum response) of the $\mathrm{A}_{0}$ mode has been predicted around $60 \mathrm{kHz}$ and experimentally identified around

Q21 $50 \mathrm{kHz}$, whereas $\mathrm{A}_{0}$-mode rejection has been predicted around $190 \mathrm{kHz}$, which seems reasonably close to the experimental trend. Examination of Fig. $11 \mathrm{~b}$ indicates that a reasonable match between theory and experiment has been achieved in this initial work; however, further extensive studies are necessary to develop a full description of this tuning and rejection phenomenon for various composite layups and various wave propagation and directions. In this work, the theoretical predictions were done in the frequency domain with single frequency excitation; future work should also address a comparison between frequency domain and time domain identification.

\section{Experimental Results of Damage Detection in Composite Plates}

We did a series of experiments to detect damage in composite plates [26]. The first type of damage we used in our experiments was a small hole of increasing diameter. Holes are generally not a representative type of damage for composite structures; however, we decide to use holes first in our damage detection tests, because this type of damage can be easily manufactured and reproduced with accuracy. The second type of damage we considered in our experiments was impact damage. This type of damage was produced using an inertial impactor. Details of these two types of experiments are given in the following two sections.

\section{A. Hole Damage Detection in Unidirectional Composite Specimens}

We performed experiments to detect small holes of increasing size in unidirectional composite strips. Two unidirectional strips $(41 \mathrm{~cm}$ by $5 \mathrm{~cm}$ ) were used for these experiments. Both strips we instrumented with two $7 \mathrm{~mm}$ round PWASs placed $150 \mathrm{~mm}$ apart (Fig. 12). The PWAS transducers were used in pitch-catch mode. In one experimental setup, the hole damage was placed directly in the pitch-catch path; since this placement is the most favorable for detection, we used this experiments to determine the detection threshold: i.e., the smallest detectable hole size. In the second experimental setup, we placed the hole offset by $20 \mathrm{~mm}$ from the pitch-catch path. Again, we performed detection experiments with holes of increasing size in order to determine the smallest detectable hole size in this less favorable condition. The hole-size diameters are given in Table 1 .

The first readings were taken when the strips were undamaged (baselines). Then, we drilled a $0.8 \mathrm{~mm}$ hole on both, and we enlarged them in 11 steps till they reached $6.35 \mathrm{~mm}$ in diameter. Table 1 reports the dimension of the holes for each step and reading. All readings

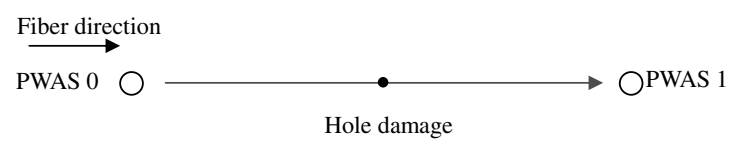

a)

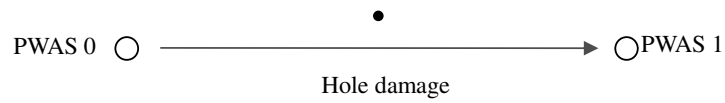

b)

Fig. 12 Unidirectional composite strips with PWASs installed $150 \mathrm{~mm}$ apart. a) hole damage placed on the pitch-catch path; b) hole damaged placed $20 \mathrm{~mm}$ offset from the pitch-catch path.
Table 1 Hole-size diameters used in damage detection experiments on unidirectional composite strips

\begin{tabular}{ccc}
\hline \hline Step & Readings & Hole size, $\mathrm{mm}$ \\
\hline 0 & $00-04$ & - \\
1 & $05-09$ & 0.8 \\
2 & $10-14$ & 1.5 \\
3 & $15-19$ & 1.6 \\
4 & $20-25$ & 2.0 \\
5 & $26-30$ & 2.4 \\
6 & $31-35$ & 3.2 \\
7 & $36-40$ & 3.6 \\
8 & $41-45$ & 4.0 \\
9 & $46-50$ & 4.8 \\
10 & $51-55$ & 5.5 \\
11 & $56-60$ & 6.4 \\
\hline \hline
\end{tabular}

were recorded at $480 \mathrm{kHz}$. At this frequency we have a single strong $S_{0}$ wave packet.

During the pitch-catch experiments, a three-count smoothed toneburst was applied at the transmitter PWAS-0; the traveling-wave packet was measured at the receiver PWAS-1. The tone-burst frequency was $480 \mathrm{kHz}$; this frequency was selected because, at this frequency, we had a strong quasi- $\mathrm{S}_{0}$ wave packet (tuning effect). The first pitch-catch reading was taken with the strip in pristine (undamaged) condition. This reading was used as baseline. Five separate consecutive readings were taken in order to achieve a degree of statistical variation for our experiment. Then, a $0.8 \mathrm{~mm}$ hole was drilled and another battery of five pitch-catch readings was taken. The process was repeated with ever increasing hole sizes. Thus, 11 reading steps were obtained until the hole reached a $6.35 \mathrm{~mm}$ diameter.

The collected data were analyzed with the damage index (DI) method. The DI value was computed with the root-mean-square deviation (RMSD) algorithm: i.e.,

$$
\operatorname{RMSD}=\sqrt{\sum_{N}\left[\operatorname{Re}\left(u_{i}\right)-\operatorname{Re}\left(u_{i}^{0}\right)\right]^{2} / \sum_{N}\left[\operatorname{Re}\left(u_{i}^{0}\right)\right]^{2}}
$$

where $N$ is the number of points in the analyzing window; $u_{i}$ is the current reading; $u_{i}^{0}$ is the baseline reading.

The resulting DI values were plotted (Fig. 13). The detection results for the case when the hole is directly in the pitch-catch path are given in Fig. 13a, and the detection results for the hole placed offside from the pitch-catch path are given in Fig. 13b. The first five readings were for the baseline: i.e., for the pristine specimen without damage. The next five readings are for the specimen with the $0.8 \mathrm{~mm}$ hole, and so on. If we look at Fig. 13a representing the results for the damage in the direct pitch-catch path, we note that as soon as damage was inflicted on the specimen, the DI value changed. This indicates that the detection method is very sensitive to small damage. The next three groups on the plot in Fig. 13a give the DI values for hole sizes of $0.8,1.5$, and $1.6 \mathrm{~mm}$. We note that the change from 0.8 to $1.5 \mathrm{~mm}$ damage size produced a clear jump in the DI value, but the next two changes were much smaller; this seems to be consistent with the fact that the relative change from 1.5 to $1.6 \mathrm{~mm}$ is much smaller than from 0.8 to $1.5 \mathrm{~mm}$. The DI values keep increasing with increasing hole size; the only anomaly is that no change was registered by the DI when the hole size increased from 2 to $2.4 \mathrm{~mm}$. Note that the biggest jumps in DI values (from reading 20 to reading 21 and from reading 45 to reading 46 ) were achieved for the biggest changes in hole size.

Figure $13 \mathrm{~b}$ gives the DI results for the hole damage placed offside from the pitch-catch path. The DI value increased as soon as damage was inflicted. An unexpected fact happened when the hole size was increased from 2.0 to $2.4 \mathrm{~mm}$ : in this case, the DI increase was much larger than before; we suspect that some additional unintended damage was produced by the drilling process, or that a threshold in the interaction between the guided waves and the hole damage was reached; further investigation of this phenomenon is warranted, but 


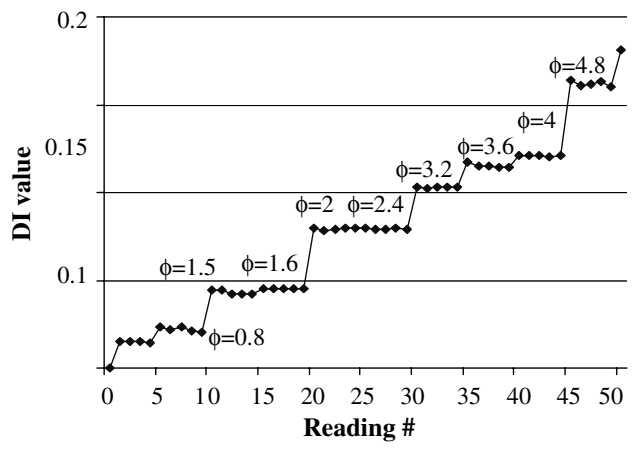

a)

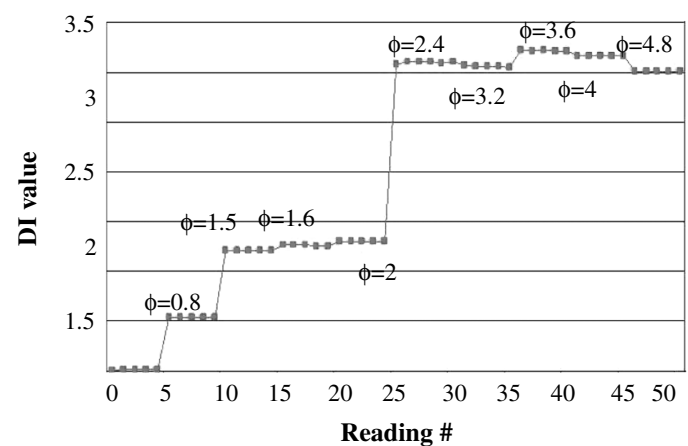

b)

Fig. 13 DI analysis of damage detection in an unidirectional composite strip using the pitch-catch method: a) hole damage placed in the pitch-catch path; b) hole damaged placed offside from the pitch-catch path. Each group of five readings represents five consecutive readings taken at the same damage level (hole size); the hole-size values are given in Table 1.

could not be achieved in the time and funding framework of the reported project. It is also interesting and curious to note that in Fig. 13, the DI for the smallest offside hole is much larger than that even for the largest hole directly in the pitch-catch path! This aspect warrants further investigation, because it is not clear at this stage if this reflects a poor metric of if it is a peculiarity of pitch-catch approach. At first sight, this observation would suggest that the methodology is a poor indicator of damage size, since just a $2 \mathrm{~cm}$ offset in damage location from the direct path could throw the metric off significantly. It is our intention to investigate this phenomenon further and report it in a future publication.

Overall, we can conclude that these initial experiments have shown that the pitch-catch method can detect hole damage in an unidirectional composite. In addition, these experiments have shown that the damage detection is also possible when the damage is placed offside from the pitch-catch path. This latter fact is explainable through the fact that guided waves are diffracted by the damage even when the damage is not placed directly in the pitch-catch path. Further work is warranted to continue this investigation and clarify some of the results.

\section{B. Damage Detection in a Quasi-Isotropic Composite Plate}

In this set of experiments, we used a 1240 by $1240 \mathrm{~mm}$ quasiisotropic plate with a $[(0 / 45 / 90 /-45) 2]_{S}$ layup of T300/5208 unidirectional tape; the overall thickness was $2.25 \mathrm{~mm}$. Two damage types were considered: drilled holes of increasing diameter and impact created with an inertial impactor. The experimental setup is shown in Fig. 14. The impact locations are marked as 1 and 2. A set of 12 PWAS transducers were installed in pairs, as shown in Fig. 14. The PWAS pairs were (p0-p1), (p2-p3), (p4-p5), (p8-p9), (p10$\mathrm{p} 11)$, and (p12-p13). The distance between the PWAS pairs was $300 \mathrm{~mm}$. The excitation signal was a three-count $11 \mathrm{~V}$ smoothed tone burst. The data were collected automatically using an ASCU2 signal switch (Fig. 14). We collected data from PWASs p0, p1, p5, p8, p12, and p13. Each PWAS was, in turn, the transmitter and the receiver. Three frequency values were used: $f=54 \mathrm{kHz}$ when only the $\mathrm{A}_{0}$ mode was present, $f=225 \mathrm{kHz}$ when only the $\mathrm{S}_{0}$ mode was present, and $f=255 \mathrm{kHz}$ when the $\mathrm{S}_{0}$ mode had maximum amplitude. Four sequential baseline readings were taken with the plate undamaged. Subsequent readings were taken after each damage type was applied to the plate.

\section{Hole Damage Detection in a Quasi-Isotropic Composite Plate}

A hole of increasing size was drilled between PWASs p1 and p12 (see Fig. 14). The location of the hole was halfway between these two PWASs. The diameter of the hole was increased in 14 steps using the drill sizes shown in Table 2 . At each damage step, several readings were taken. Table 2 shows the step index number, the reading index numbers for that step, and the hole drill size. Data processing consisted in comparing each reading with the baseline (reading 00) and calculating the damage index (DI). The DI value was computed

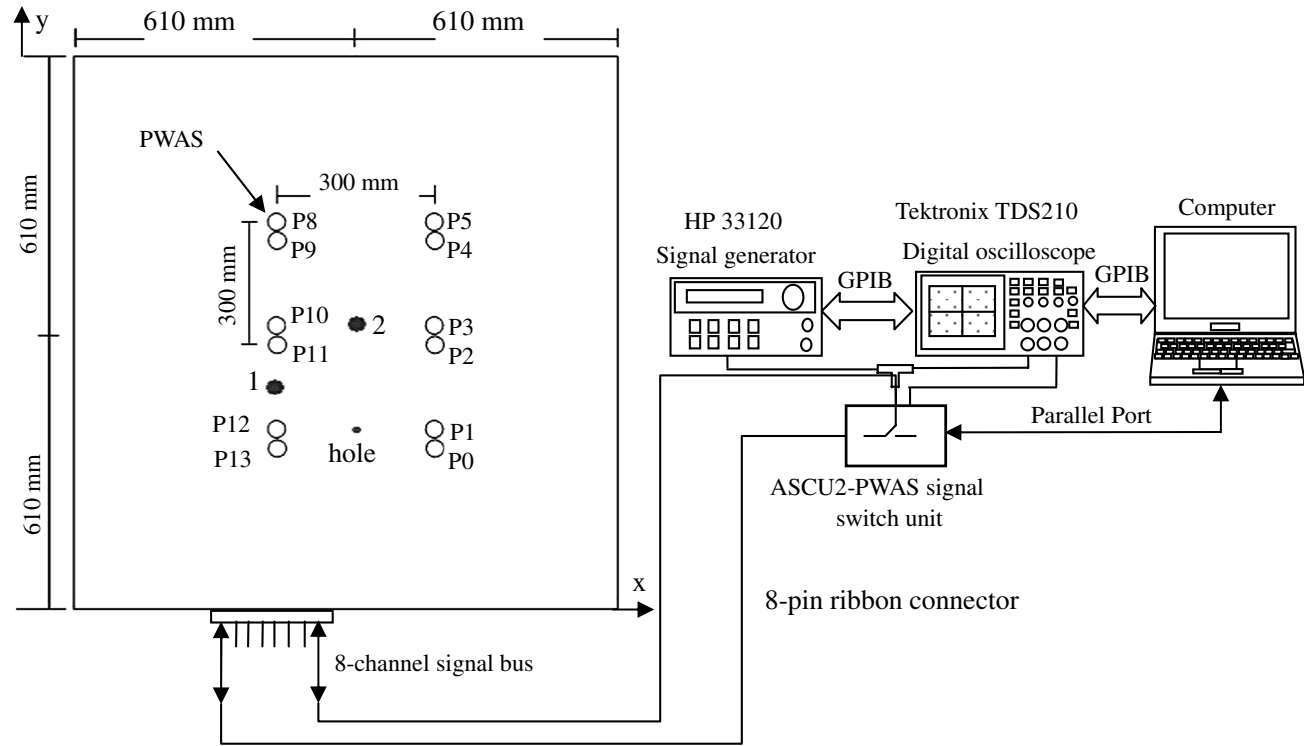

Fig. 14 Experimental setup for hole-damage detection on quasi-isotropic composite panel. Featured on the plate are 14 PWAS transducers (p0 through p13), one hole-damage location, and two impact locations (1 and 2). 
Table 2 Hole diameters for the damage detection experiments on quasi-isotropic composite panel

\begin{tabular}{ccll}
\hline \hline & & \multicolumn{2}{c}{ Hole size } \\
\cline { 3 - 4 } Step & Readings & mil & $\mathrm{mm}$ \\
\hline 1 & $00-03$ & 0 & - \\
2 & $04-07$ & 032 & 0.81 \\
3 & $08-11$ & 059 & 1.50 \\
4 & $12-15$ & 063 & 1.60 \\
5 & $16-19$ & 078 & 1.98 \\
6 & $20-23$ & 109 & 2.77 \\
7 & $24-28$ & 125 & 3.18 \\
8 & $29-32$ & 141 & 3.58 \\
9 & $33-36$ & 156 & 3.96 \\
10 & $37-40$ & 172 & 4.37 \\
11 & $41-44$ & 188 & 4.78 \\
12 & $45-48$ & 203 & 5.16 \\
13 & $49-52$ & 219 & 5.56 \\
14 & $53-56$ & 234 & 5.94 \\
\hline \hline
\end{tabular}

with the RMSD algorithm. We analyzed the DI data with statistical software (SAS) and stated our conclusions to a significance of $99 \%$.

a. Pitch-Catch Analysis. For pitch-catch analysis, we took into consideration only the data coming from the following PWAS pairs: 1) PWAS p0 transmitter and PWAS p13 receiver, 2) PWAS p1 transmitter and PWAS p12 receiver, and 3) PWAS p5 transmitter and PWAS p8 receiver.

This experiment determined the minimum hole size that two PWAS pairs (p0-p13, p1-p12) were able to detect. We also used PWAS pair p5-p8, which was far away from the hole damage and hence nominally insensitive to damage. This allowed us to verify that

Q23 our method is consistent and does not give false positives; i.e., no detection comes out when not supposed to do.

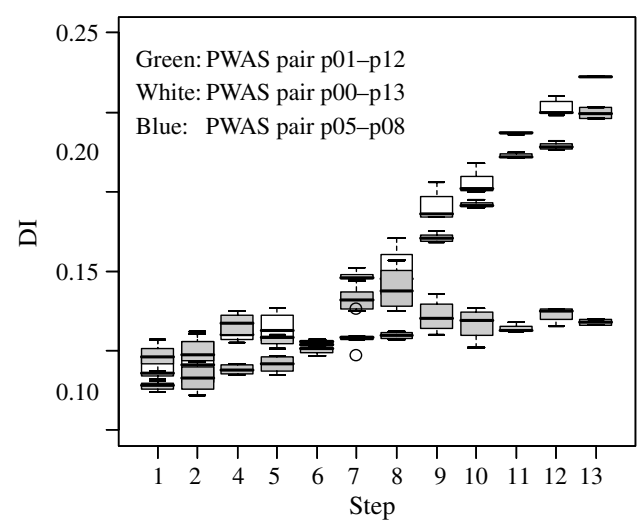

a)

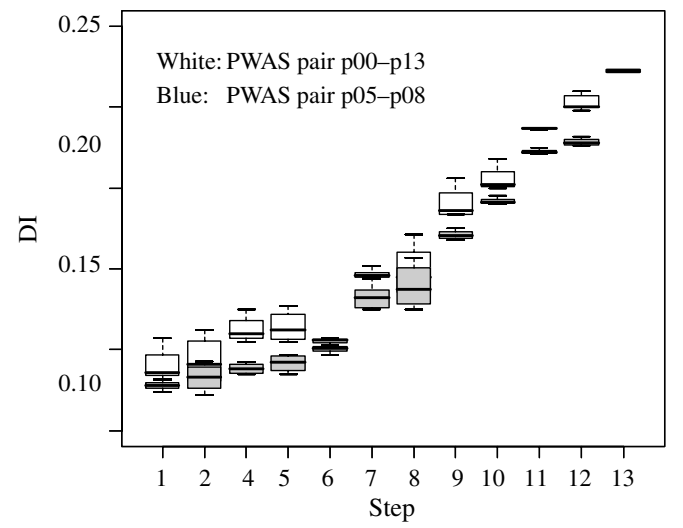

Fig. 15 Pitch-catch hole-detection results showing DI values at different damage step values and different PWAS pairs: a) $f=54 \mathrm{kHz}$ (i.e., when only $A_{0}$ mode is present), b) $f=225 \mathrm{kHz}$ (i.e., when only $S_{0}$ mode is present), and c) $f=255 \mathrm{k} \mathrm{Hz}$ (i.e., when the $\mathrm{S}_{0}$ mode has maximum amplitude.

Figure 15a shows the $54 \mathrm{kHz}$ detection results; at $54 \mathrm{kHz}$, only $\mathrm{A}_{0}$ mode was present. The wave velocity is $1580 \mathrm{~m} / \mathrm{s}$; the wavelength is $29.3 \mathrm{~mm}$. Figure 15a shows the box plot of the DI values for the three different PWAS pairs. As the hole diameter increases, the DI values for the two PWAS pairs close to the hole (p0-p13 and p1-p12) increase, whereas the DI values for the PWAS pair p5-p8 remain almost constant. We analyzed the data with statistical software (SAS), and we observed that with a significance of $99 \%$, PWAS pair p1-p12 can detect the presence of a hole with at least $2.77 \mathrm{~mm}$ diameter. On the other hand, the PWAS pair $\mathrm{p} 0-\mathrm{p} 13$ could detect the presence of a hole with the same $99 \%$ significance only when the hole diameter was at least $3.18 \mathrm{~mm}$. The DI for the PWAS pair p05-p08 did not show any significant change, as expected.

Figure $15 \mathrm{~b}$ shows the $225 \mathrm{kHz}$ detection results. At $225 \mathrm{kHz}$, only $\mathrm{S}_{0}$ mode was present. The wave velocity is about $6000 \mathrm{~m} / \mathrm{s}$, the wavelength is $26.6 \mathrm{~mm}$. Figure $15 \mathrm{~b}$ shows the box plot of the DI values for the three different PWAS pairs. As the hole diameter increases, the DI values for the two PWAS pairs close to the hole (p0p13 and p1-p12) increase, whereas the DI value for the PWAS pair p05-p08 remains almost the same. We conclude that the PWAS pairs p0-p13 and p1-p12 could detect the hole damage with a significance of $99 \%$ when the hole diameter reaches $2.77 \mathrm{~mm}$. On the other hand, the PWAS pair p5-p8 did not detect damage, as expected.

Figure $15 \mathrm{c}$ shows the $255 \mathrm{kHz}$ detection results. At $225 \mathrm{kHz}$, the $\mathrm{S}_{0}$ mode has maximum amplitude. The wave velocity is about $6000 \mathrm{~m} / \mathrm{s}$, the wavelength is $23.5 \mathrm{~mm}$. Figure $15 \mathrm{c}$ shows the box plot of the DI values for the three different PWAS pairs. As the hole diameter increases, the DI values for the two PWAS pairs close to the hole (pairs p0-p13 and p1-p12) increases, whereas the DI for the PWAS pair $\mathrm{p} 5-\mathrm{p} 8$ remains almost constant. We conclude that with a significance of $99 \%$, the PWAS pairs p0-p13 and p1-p12 could detect the presence of the hole when its diameter was at least $3.18 \mathrm{~mm}$. On the other hand, the PWAS pair p5-p8 did not detect damage, as expected.

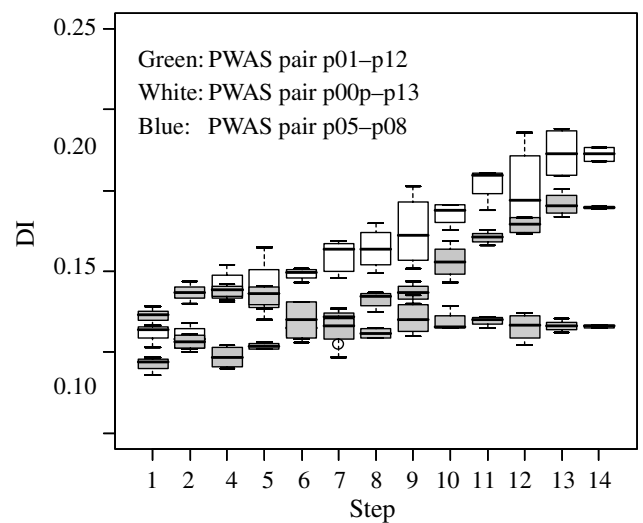

b) 
b. Pulse-Echo Analysis. Pulse-echo analysis was performed for PWASs $\mathrm{p} 0-\mathrm{p} 1$. PWAS $\mathrm{p} 0$ was used as the transmitter, and PWAS p1 was used as the receiver. Here, we report the analysis only for $f=54 \mathrm{kHz}$ when only the $\mathrm{A}_{0}$ mode is present; however, similar results were obtained at the other two frequencies. Figure 16 shows the DI box plot at different damage values. Analyzing the data we find that the first significant difference with respect to step 1 (baseline) appears at step 7, corresponding to a hole of $3.18 \mathrm{~mm}$. Hence, we concluded that with $99 \%$ confidence, the pulse-echo method could detect a hole with a size of at least $3.18 \mathrm{~mm}$.

This set of hole-detection experiments indicated that PWAS transducers used in pitch-catch and pulse-echo modes are able to detect holes in a 2.25-mm-thick isotropic composite plate. The minimum hole size detected with $99 \%$ confidence was $2.77 \mathrm{~mm}$.

\section{Impact Damage Detection in a Quasi-Isotropic Composite Plate}

The second type of damage considered for detection in the quasiisotropic composite plate was the damage caused by a low-velocity impact. This type of damage was produced with the impactor equipment shown in Fig. 17. The impactor had a hemispherical tip of $12.7 \mathrm{~mm}$ diameter $(0.5 \mathrm{in}$.). Its weight was $391 \mathrm{~g}(13.79 \mathrm{oz})$. The

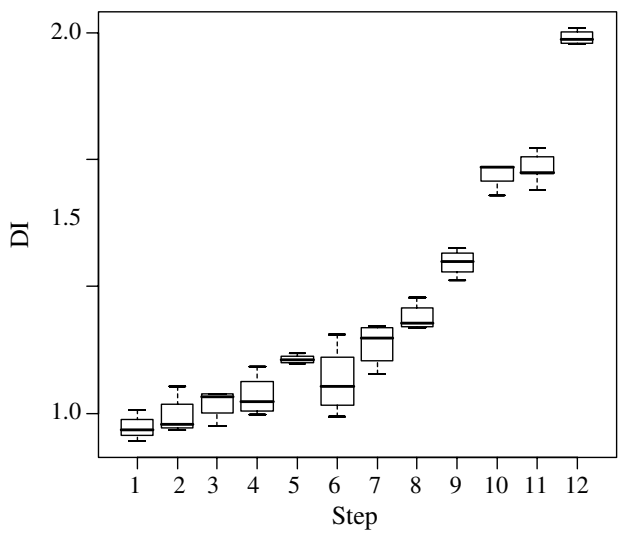

Fig. 16 Pulse-echo hole-detection results showing DI values at different damage step values and different PWAS pairs for $f=$ $54 \mathrm{kHz}$ (i.e., when only $A_{0}$ mode is present).
Table 3 Synopsis of the impacts applied to the composite plate of Fig. 14

\begin{tabular}{|c|c|c|c|c|c|}
\hline \multirow[b]{2}{*}{ Readings } & \multicolumn{2}{|c|}{ Energy } & \multicolumn{2}{|c|}{ Velocity } & \multirow[b]{2}{*}{ Step } \\
\hline & $\mathrm{N} \cdot \mathrm{m}$ & $\mathrm{ft} \cdot \mathrm{lb}$ & $\mathrm{m} / \mathrm{s}$ & $\mathrm{ft} / \mathrm{s}$ & \\
\hline & \multicolumn{3}{|c|}{ Damage A } & & \\
\hline $00-10$ & & & & $\overline{ }$ & 1 \\
\hline $11-20$ & 8 & 6 & 3.4 & 11.2 & 2 \\
\hline \multirow[t]{2}{*}{$21-30$} & 16 & 12 & 3.4 & 16.0 & 3 \\
\hline & \multirow{2}{*}{\multicolumn{3}{|c|}{ Damage B }} & & \\
\hline 00-10 & & & & $\overline{140}$ & 1 \\
\hline $11-20$ & 8 & 6 & 4.2 & 14.0 & 2 \\
\hline $21-30$ & 16 & 12 & 5.9 & 19.8 & 3 \\
\hline
\end{tabular}

impactor weight could be increased by adding barrels (Fig. 17b) to the base configuration of Fig. 17a. Each barrel weighed 500 g. $(1 \mathrm{lb}$, $1.63 \mathrm{oz}$ ); a total of three barrels could be assembled on the impactor.

Two impact damages were produced on the plate with two different impactor configurations (respectively, different weights and different number of barrels). The impactor used for damage $\mathrm{A}$ had a

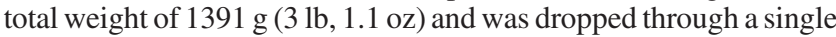
barrel. The first impact had an energy level of $6 \mathrm{ft} \cdot \mathrm{lb}$ and hit the plate at about $3.42 \mathrm{~m} / \mathrm{s}(11.22 \mathrm{ft} / \mathrm{s})$; the second impact had an energy level of $12 \mathrm{ft} \cdot \mathrm{lb}$ and hit the plate at about $4.84 \mathrm{~m} / \mathrm{s}(15.87 \mathrm{ft} / \mathrm{s})$. The impactor used for damage B had a total weight of $891 \mathrm{~g}(1 \mathrm{lb}, 15.5 \mathrm{oz})$ The first impact had an energy level of $6 \mathrm{ft} \cdot \mathrm{lb}$ and hit the plate at about $4.28 \mathrm{~m} / \mathrm{s}(14.03 \mathrm{ft} / \mathrm{s})$; the second impact had an energy level of $12 \mathrm{ft} \cdot \mathrm{lb}$ and hit the plate at about $6 \mathrm{~m} / \mathrm{s}(19.83 \mathrm{ft} / \mathrm{s})$.

Table 3 reports the energy and velocity levels for the two damage cases. For both damage A and damage B we recorded 11 baseline readings and 10 readings for each energy level. The readings were collected through the ASCU2 system.

The first impact (damage A) was produced between PWAS 12 and PWAS 11 (marked as 1 in Fig. 14). No visual damage was produced at $6 \mathrm{ft} \cdot \mathrm{lb}$ energy level. After the second impact at energy level of $12 \mathrm{ft} \cdot \mathrm{lb}$, damage could be seen on the opposite side of the plate. We took readings for PWAS pairs p11-p12 and p9-p10. Each PWAS transducer in each pair was used once as the transmitter and once as the receiver.

The second impact damage (damage B) was produced between PWAS p2 and PWAS p11 (marked as 2 in Fig. 14). We collected

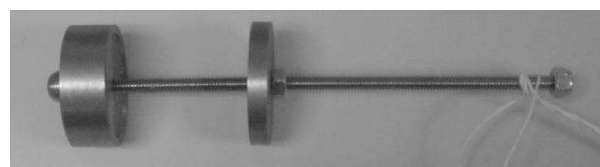

a)

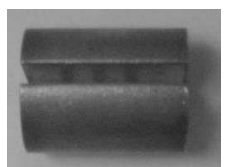

b)

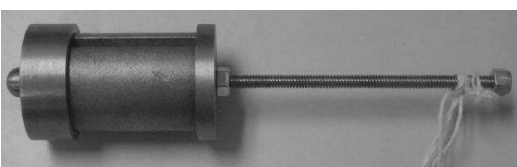

c)

Fig. 17 Impactor equipment used to generate impact damage in the quasi-isotropic specimen: a) impactor with hemispherical tip, b) barrel, and c) impactor assembly.
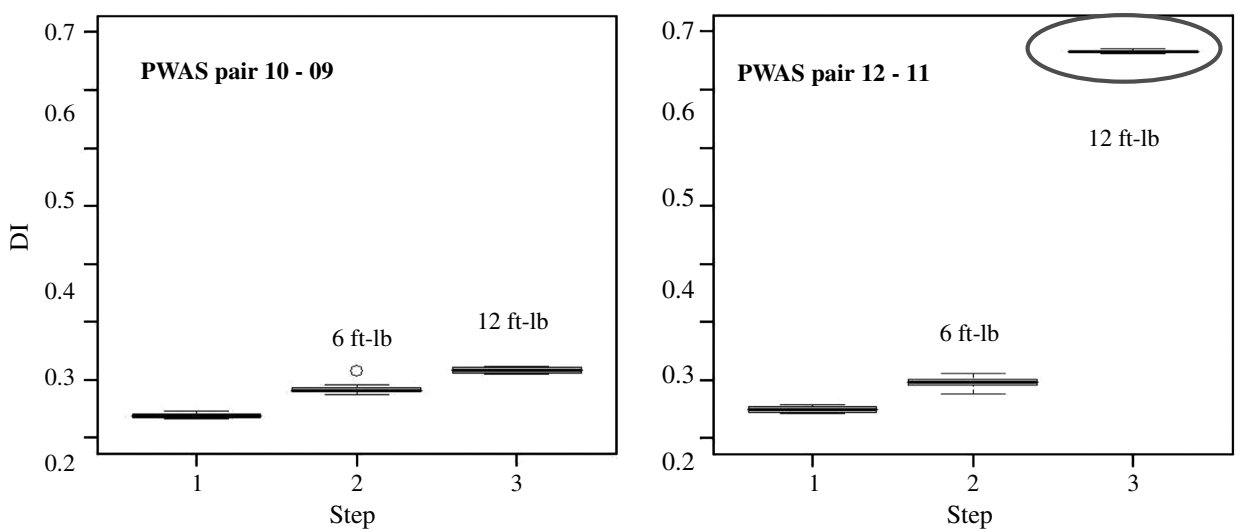

Fig. $18 \mathrm{~A}_{0}$-mode detection of impact damage A: DI values as a function of the damage level for PWAS pairs p9-p10 and p11-p12 $(f=54 \mathrm{kHz})$ 

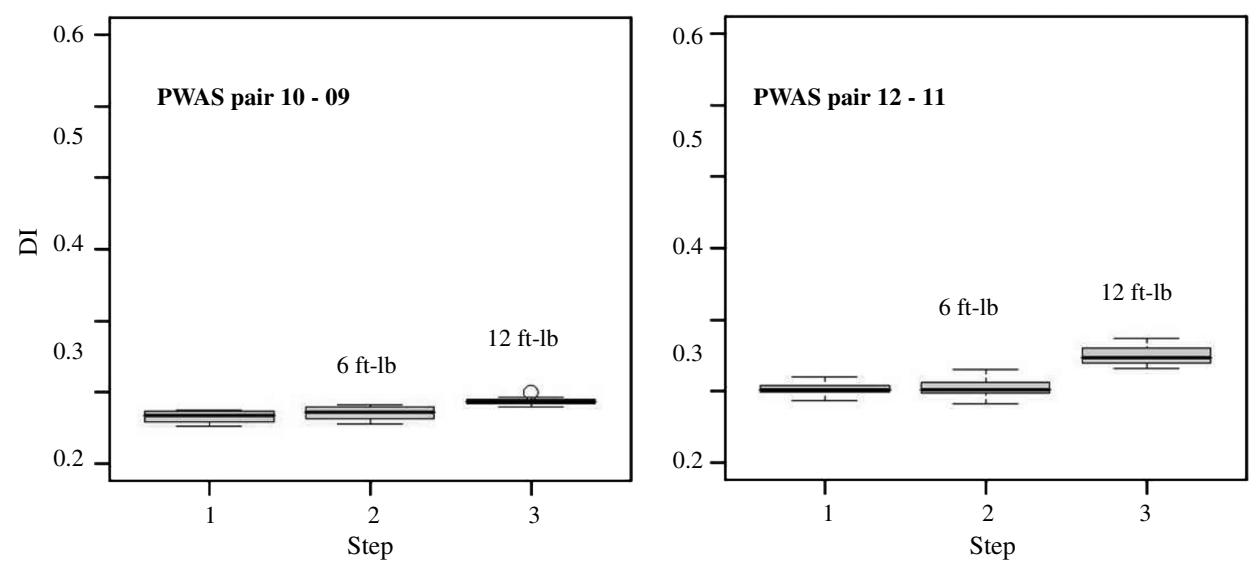

Fig. $19 \mathrm{~S}_{0}$-mode detection of impact damage A: DI values as a function of the damage level for PWAS pairs p9-p10 and p11-p12 $(f=225 \mathrm{kHz})$.

readings from PWAS pairs p2-p11, p3-p10, and p5-p8. During the experiment, each PWAS was once the transmitter and once the receiver. No visible damage was produced after the two impacts. However, the presence of damage in the plate structure was registered with standard ultrasonic methods.

a. Pitch-Catch Analysis. Pitch-catch analysis was done at two frequencies: $f=54 \mathrm{kHz}$ (with only the $\mathrm{A}_{0}$ mode present) and $f=225 \mathrm{kHz}$ (with only the $\mathrm{S}_{0}$ mode present). Figure 18 shows the $\mathrm{A}_{0}$ DI values for damage A (marked as 1 in Fig. 14). There is not significant change between the DI values of the PWAS pair $\mathrm{p} 9-\mathrm{p} 10$, which are far away from the impact damage. However, for the PWAS pair p11-p12, which lies on either side of damage A location, there is significant change after the second impact (energy level $12 \mathrm{ft} \cdot \mathrm{lb}$ ). Similar results were obtained for damage B with PWAS pairs $\mathrm{p} 2$ $\mathrm{p} 10, \mathrm{p} 3-\mathrm{p} 10$, and $\mathrm{p} 5-\mathrm{p} 8$. This analysis indicates that it is possible to detect the damage after the second stronger impact. Figure 19 shows the $S_{0}$ DI values for damage A. As before, the PWAS pair $\mathrm{p} 9-\mathrm{p} 10$ that is far from the impact damage does not show much change in the DI values. The PWAS pair p11-p12 that is placed on either side of the impact damage shows a DI change after the second impact (energy level $12 \mathrm{ft} \cdot \mathrm{lb}$ ), but the change is not as clear as in the case of $\mathrm{A}_{0^{-}}$ mode detection. We conclude that the pseudo $S_{0}$ mode is less sensitive to impact damage in a quasi-isotropic composite panel. Similar results were obtained for damage B with PWAS pairs $\mathrm{p} 2$ p10, p3-p10, and p5-p8.

b. Pulse-Echo Analysis. Pulse-echo analysis was performed for PWASs p10 and p11 (damage A). PWAS p11 was used as the transmitter, and PWAS p10 was used as the receiver. We report the analysis for the $\mathrm{A}_{0}$ mode $(54 \mathrm{kHz})$ and $\mathrm{S}_{0}$ mode $(225 \mathrm{kHz})$. The results are shown in Fig. 20. Analysis of the data indicates that there is significant difference between step 1 (baseline) and the damage steps 2 and 3 for the case of $\mathrm{A}_{0}$ waves $\left(54 \mathrm{kHz}\right.$ ). For the case of $\mathrm{S}_{0}$ waves $(225 \mathrm{kHz})$, there is significant difference only between step 1 and step 3 (impact at $12 \mathrm{ft} \cdot \mathrm{lb}$ ). This indicates that, as in the pitchcatch method, the $S_{0}$ mode is less sensitive to impact damage.

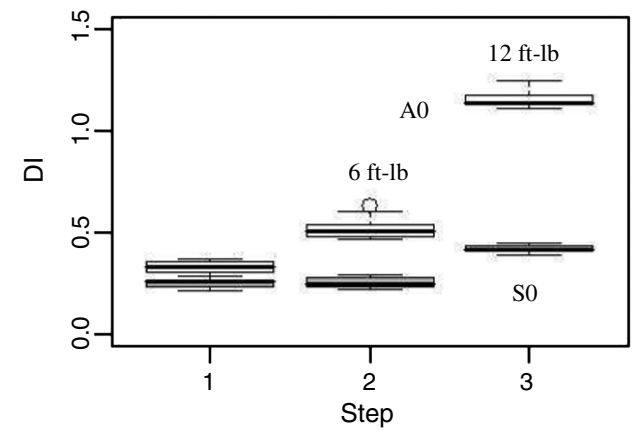

Fig. 20 Damage A DI values at different step values and frequency $54 \mathrm{kHz}$ (white box) and $225 \mathrm{kHz}$ (gray box).

\section{Conclusions}

This paper has presented how piezoelectric-wafer active sensors (PWASs) can be used to detect damage in composite structures. The guided-wave propagation in composites and PWAS tuning effects were analyzed both theoretically and experimentally. Reasonable agreement was found between the predictive theory presented in the paper and the tuning experiments on actual composite specimens. Subsequently, the paper presented experimental damage detection results in composites: hole damage in unidirectional and quasiisotropic plates and impact damage in quasi-isotropic plates. In unidirectional carbon-epoxy composite strips, it was found that the minimum detectable hole size was $0.8 \mathrm{~mm}$. The detection method used in these experiments was pitch-catch with $480 \mathrm{kHz}$ tuned $\mathrm{S}_{0}$ waves. In quasi-isotropic carbon-epoxy composite plates, it was found that the minimum detectable hole size with $99 \%$ confidence was $2.77 \mathrm{~mm}$. In these experiments, we used several tuned guidedwave modes: $A_{0}$ waves at $54 \mathrm{kHz}$ and $\mathrm{S}_{0}$ waves at 225 and $255 \mathrm{kHz}$. The detection methods used in these experiments were both pitchcatch and pulse-echo. In the impact damage experiments, it was found that tuned $\mathrm{A}_{0}$ guided-wave modes were much more effective in detecting impact damage in quasi-isotropic carbon-epoxy plates than $\mathrm{S}_{0}$ modes.

However, the above conclusions about confidence levels and minimum detectable damage size should be interpreted in the appropriate context, since just one specimen was used for each of the tests, with one set of specific transducer sizes, with damage along one or two directions at particular distances. In practice, composite plates show greater manufacturing variability from panel to panel when compared with metals. It should be borne in mind that the experimental results presented form a small data set and that these are preliminary, limited, conclusions. Further research needs to be done to better understand the interaction of guided waves with damage in composite materials and how various guided-wave types interact with various types of damage. An estimation of variability should be achieved by testing of a larger set of specimens in correlation with appropriate predictive modeling. Of particular interest should also be an estimation of the detection range and directionality effects, which make guided-wave methods more difficult to handle in composites than in metals, given that composites have nonnegligible internal damping and present significant steering characteristics.

\section{References}

[1] Rose, J. L., and Soley, L., "Ultrasonic Guided Waves for the Detection of Anomalies in Aircraft Components," Materials Evaluation, Vol. 50, No. 9, 2000, pp. 1080-1086.

[2] Sohn, H., Farrar, C. R., Hemez, F. M., Shunk, D. D., Stinemates, S. W., Nadler, B. R., and Czarnecki, J. J., "A Review of Structural Health Monitoring Literature from 1996-2001," Los Alamos National Lab., Rept. LA-13976-MS, Albuquerque, NM, 2004.

[3] Giurgiutiu, V., Zagrai, A., and Bao, J., "Damage Identification in Aging Aircraft Structures with Piezoelectric Wafer Active Sensors," Journal of 
Intelligent Material Systems and Structures, Vol. 15, Nos. 9-10, 2004 pp. 673-687.

doi: $10.1177 / 1045389 X 04038051$

[4] Soutis, C., and Beaumont, P. W. R., Multi-Scale Modelling of Composite Material Systems: The Art of Predictive Damage Modelling, Woodhead, Sawston, England, U.K., 2005.

[5] Soutis, C., "Recent Advances in Building with Composites," Plastics, Rubber and Composites: Macromolecular Engineering, Vol. 38, Nos. 9-10, 2009, pp. 359-366. doi:10.1179/146580109X12540995045606

[6] Diamanti, K., Soutis, C., and Hodgkinson, J. M., "Non-Destructive Inspection of Sandwich and Repaired Composite Laminated Structures," Composites Science and Technology, Vol. 65, No. 13, 2005, pp. 2059-2067. doi:10.1016/j.compscitech.2005.04.010

[7] "Aircraft Structures," U.S. Dept. of Defense, Joint Service Specification Guide JSSG-2006, 30 Oct. 1998.

[8] "Aircraft Structural Integrity Program, General Guidelines for," U.S. Dept. of Defense MIL-HDBK-1530, 4 Nov. 1996.

[9] Kashtalyan, M., and Soutis, C., "Analysis of Composite Laminates with Intra- and Interlaminar Damage," Progress in Aerospace Sciences, Vol. 41, No. 2, 2005, pp. 152-173. doi:10.1016/j.paerosci.2005.03.004

[10] Soutis, C., and Guz, I. A., "Fracture of Layered Composites by Internal Fibre Instability: Effect of Interlaminar Adhesion," The Aeronautical Journal, Vol. 110, No. 1105, 2006, pp. 185-195.

[11] Soutis, C., "Modelling the Open Hole Compressive Strength of Composite Laminates Tested in Hot-Wet Conditions," Plastics, Rubber and Composites Processing and Applications, Vol. 38, Nos. 2-4, 2009, pp. 55-60. doi: $10.1179 / 174328909 \times 387810$

[12] Lee, J. D., Kong, C. D., and Soutis, C., "Modelling of Low Velocity Impact Damage in Laminated Composites," Journal of Mechanical Science and Technology, Vol. 19, No. 4, 2005, pp. 947-957. doi:10.1007/BF02919177

[13] Cawley, P., "The Rapid Non-Destructive Inspection of Large Composite Structures," Composites, Vol. 25, No. 5, 1994, pp. 351-357. doi:10.1016/S0010-4361(94)80005-7

[14] Bar-Cohen, Y., "In-Service NDE of Aerospace Structures-Emerging Technologies and Challenges at the End of the 2nd Millennium," NDT.net [online journal], Vol. 4, No. 9, 1999, pp. 1-21, http:// www.ndt.net/article/v04n09/bcohen/bcohen.htm [retrieved 2010].

[15] Giurgiutiu, V., Structural Health Monitoring with Piezoelectric Wafer Active Sensors, Elsevier, New York, 2008, pp. 239-588.

[16] Giurgiutiu, V., Yu, L., Kendall, J., and Jenkins, C., "In Situ Imaging of Crack Growth with Piezoelectric Wafer Active Sensors," AIAA Journal,
Vol. 45, No. 11, Nov. 2007, pp. 2758-2769. doi: $10.2514 / 1.30798$

[17] Rose, J. L., Ultrasonic Waves in Solid Media, Cambridge Univ. Press, Cambridge, England, U.K., 1999, pp. 177-200.

[18] Wilcox, P., Lowe, M., and Cawley, P., "Lamb and SH Wave Transducer Arrays for the Inspection of Large Areas of Thick Plates," Review of Progress in Quantitative Nondestructive Evaluation, edited by D. O. Thompson, and D. E. Chimenti, Plenum, New York, 2000, pp. 1049 1056.

[19] Birt, E. A., "Damage Detection in Carbon-Fibre Composites Using Ultrasonic Lamb Waves," Insight, Vol. 40, No. 5, 1998, pp. 335-339.

[20] Diamanti, K., Soutis, C., and Hodgkinson, J. M., "Piezoelectric Transducer Arrangement for the Inspection of Large Composite Structures," Composites, Part A: Applied Science and Manufacturing, Vol. 38, No. 4, 2007, pp. 1121-1130. doi:10.1016/j.compositesa.2006.06.011

[21] Knopoff, L., "A Matrix Method for Elastic Wave Problems," Bulletin of the Seismological Society of America, Vol. 54, No. 1, Feb. 1964, pp. 431-438.

[22] Thomson, W. T., "Transmission of Elastic Waves Through a Stratified Solid Medium," Journal of Applied Physics, Vol. 21, 1950, pp. 89-93. doi:10.1063/1.1699629

[23] Haskell, N. A., "Dispersion of Surface Waves on Multilayered Media," Bulletin of the Seismological Society of America, Vol. 43, 1953, pp. 17 34.

[24] Kausel, E., "Wave Propagation in Anisotropic Layered Media," International Journal for Numerical Methods in Engineering, Vol. 23, 1986, pp. 1567-1578.

doi:10.1002/nme.1620230811

[25] Nayfeh, A. H., Wave Propagation in Layered Anisotropic Media with Application to Composites, Elsevier, New York, 1995, p. 117.

[26] Santoni-Bottai, G., "Fundamental Studies in the Lamb-Wave Interaction Between Piezoelectric Wafer Active Sensor and Host Structure During Structural Health Monitoring," Ph.D. Dissertation, Univ. of South Carolina, Columbia, SC, April 2010.

[27] Rokhlin, S. I., and Wang, L., "Stable Recursive Algorithm for Elastic Wave Propagation in Layered Anisotropic Media: Stiffness Matrix Method," Journal of the Acoustical Society of America, Vol. 112, No. 3, June 2002, pp. 822-834. doi: $10.1121 / 1.1497365$

[28] Auld, A., Acoustic Fields and Waves in Solids, Vols. 1-2, Wiley, New York, 1990, p. 161. 


\section{IMPORTANT: PLEASE READ CAREFULLY.}

\section{Queries}

When production of AIAA journal papers begins, the official approved PDF is considered the authoritative manuscript. Authors are asked to submit source files that match the PDF exactly, to ensure that the final published article is the version that was reviewed and accepted by the associate editor. Once a paper has been accepted, any substantial corrections or changes must be approved by the associate editor before they can be incorporated.

If you and the EIC settled on some final changes to your manuscript after it was accepted, it is possible that your page proofs do not reflect these final changes. If that is the case, please submit these changes as itemized corrections to the proofs.

If final changes were made to the figures, please check the figures appearing in the proofs carefully. While it is usual procedure to use the figures that exist in the source file, if discrepancies are found between figures (manuscript source file vs the approved PDF), the figures from the PDF are inserted in the page proofs, again deferring to the PDF as the authoritative manuscript. If you find that agreed-upon final changes to your figures are not appearing in your page proofs, please let us know immediately.

Q1. Please confirm that the above () information is correct.

Q2. Numerous adjustments were made throughout for syntax, structure, and journal requirements. Please read closely to ensure that your meaning was retained, but please do not address changes that did not affect your meaning.

Q3. Is it correct that there are two A_k entries or is an accent missing from one?

Q4. Ellipses are not permitted in the left column of the Nomenclature and were removed from all affected entries; please add text as needed.

Q5. If you do not like the plural acronym of PWASs, please indicate what noun to uses instead in those uses: e.g., PWAS transducers

Q6. Please adjust the wording of "adequate...as...that"; would "adequate...., such as that" or "adequate...., similar to that" suit your meaning?

Q7. Please adjust the wording of "failure through...mechanisms take place"; should it be "failure, though...mechanisms take place" or "failure through...mechanisms that take place"?

Q8. Please adjust the wording of "by relative sizing the"; would "by relatively sizing the" or "by the relative sizing of the" best suit your meaning?

Q9. The word fasters was changed to fasteners here; is that correct?

Q10. For compression, the wording of "composite in three" was changed to "composite may fail under three" a) to create a complete sentence and b) for consistency with the tension wording above. Is that change correct?

Q11. The manuscript had "Error! Reference source not found." here; is there a missing citation/references?

Q12. Please adjust the wording of "are...transducers by coupling"; would "become...transducers by coupling" or "act as...transducers by coupling" suit your meaning?

Q13. Please confirm you wish to use the word "Hereunder" here, which is defined as "in accordance with this document" vs Hereafter or Next.

Q14. Please spell out SH at its lone use (or should it be SHM wave)?

Q15. a) This citation was changed from (6) to (5); is that correct? b) As discussed by e-mail, Eq. (7) was changed to (6) and all subsequent citations and equations were renumbered. Please check that all citations are accurate.

Q16. Should the space before D here be removed or is there a missing symbol or word?

Q17. Please make adjustments to cite Fig. 5 before Fig. 6.

Q18. The A0, A1, etc., were styled as $\mathrm{A}_{0}, \mathrm{~A}_{1}$, etc., for consistency; did that change retain your meaning?

Q19. Is fd correct here or should it be f_d? Are there any other uses to be changed?

Q20. ODE was spelled out at ordinary differential equation; is that correct?

Q21. The use of "A) mode" here was changed to $\mathrm{A}_{0}$ mode; $i$ that correct?

Q22. Please supply a definition for ASCU.

Q23. Please adjust the wording of "no detection comes out when not supposed to do"; would "no unexpected detection occurs" suit your meaning?

Q24. Are 05 and 08 correct here and below or should they be 5 and 8 as shown above and below?

Q25. Steps 01 and 07 were changed to 1 and 7 to match the figure and journal style.

Q26. This query was generated by an automatic reference checking system. DOI numbers for references $[1,10,12,19,21,23]$ could not be located in the databases used by the system. While the references may be correct, we ask that you check them so we can provide as many links to the referenced articles as possible.

Q27. Please check/correct the organization/publisher locations added to various references.

Q28. A check of online databases revealed a possible error in Ref. [3]. The date has been changed from '2003' to '2004'. Please confirm this is correct.

Q29. The URLs from everyspec cannot be used and were removed from [7,8].

Q30. Please confirm the URL provided for [14] and note the date you viewed it.

Q31. The references have been reordered so that they are cited in the text in numerical order.

Q32. AIAA has not indicated color use for your paper and so the color words were removed here. Please confirm that your paper should be grayscale.

Q33. What words should be substituted for green, white, and blue in Fig. 15?

Q34. Separation lines and staggered cells are not permitted in tables. In addition, the layouts went top-bottom in Table 1 but left-right in Table 2. All tables were rearranged and adjusted for journal style and consistency. Please check them for accuracy. 